ARTICLE

https://doi.org/10.1038/s41467-018-08157-y

\title{
Recurrent activating mutations of PPAR $\gamma$ associated with luminal bladder tumors
}

\author{
Natacha Rochel (1) 1, Clémentine Krucker ${ }^{2,3}$, Laure Coutos-Thévenot ${ }^{2,3}$, Judit Osz ${ }^{1}$, Ruiyun Zhang ${ }^{2,3,9}$, \\ Elodie Guyon",3, Wayne Zita ${ }^{1}$, Séverin Vanthong ${ }^{1}$, Oscar Alba Hernandez ${ }^{4}$, Maxime Bourguet ${ }^{4}$, Kays Al Badawy ${ }^{1}$, \\ Florent Dufour ${ }^{2,3}$, Carole Peluso-IItis ${ }^{1}$, Syrine Heckler-Beji ${ }^{1}$, Annick Dejaegere ${ }^{1}$, Aurélie Kamoun (D) ${ }^{5}$, \\ Aurélien de Reyniès ${ }^{5}$, Yann Neuzillet (10) ${ }^{2,3}$, Sandra Rebouissou ${ }^{2,3,10}$, Claire Béraud ${ }^{6}$, Hervé Lang ${ }^{7}$, \\ Thierry Massfelder ${ }^{8}$, Yves Allory ${ }^{2,3}$, Sarah Cianférani ${ }^{4}$, Roland H. Stote ${ }^{1}$, François Radvanyi ${ }^{2,3}$ \& \\ Isabelle Bernard-Pierrot 2,3
}

The upregulation of PPAR $\gamma / R X R \alpha$ transcriptional activity has emerged as a key event in luminal bladder tumors. It renders tumor cell growth PPAR $\gamma$-dependent and modulates the tumor microenvironment to favor escape from immuno-surveillance. The activation of the pathway has been linked to PPARG gains/amplifications resulting in PPAR $\gamma$ overexpression and to recurrent activating point mutations of RXR $\alpha$. Here, we report recurrent mutations of PPAR $\gamma$ that also activate the PPAR $\gamma / R X R \alpha$ pathway, conferring PPAR $\gamma$-dependency and supporting a crucial role of PPAR $\gamma$ in luminal bladder cancer. These mutations are found throughout the protein-including N-terminal, DNA-binding and ligand-binding domainsand most of them enhance protein activity. Structure-function studies of PPAR $\gamma$ variants with mutations in the ligand-binding domain allow identifying structural elements that underpin their gain-of-function. Our study reveals genomic alterations of PPARG that lead to protumorigenic PPAR $\gamma /$ RXR $\alpha$ pathway activation in luminal bladder tumors and may open the way towards alternative options for treatment.

\footnotetext{
${ }^{1}$ Institut de Génétique et de Biologie Moléculaire et Cellulaire (IGBMC), Institut National de La Santé et de La Recherche Médicale (INSERM), U1258/Centre National de Recherche Scientifique (CNRS), UMR7104/Université de Strasbourg, 67404 Illkirch, France. ${ }^{2}$ Institut Curie, PSL Research University, CNRS, UMR144, Equipe Labellisée Ligue contre le Cancer, 75005 Paris, France. ${ }^{3}$ Sorbonne Universités, UPMC Université Paris 06, CNRS, UMR144, 75005 Paris, France. ${ }^{4}$ Laboratoire de Spectrométrie de Masse BioOrganique, Université de Strasbourg, CNRS, IPHC UMR 7178, 67000 Strasbourg, France. ${ }^{5}$ Ligue Nationale Contre le Cancer, Programme Cartes d'Identité des Tumeurs (CIT), 75013 Paris, France. 6 UROLEAD SAS, School of Medicine, 67085 Strasbourg, France. ${ }^{7}$ Department of Urology, Nouvel Hôpital Civil Hôpitaux Universitaires de Strasbourg, Hôpitaux Universitaires de Strasbourg, 67091 Strasbourg, France. ${ }^{8}$ INSERM UMRS1113, Section of Cell Signalization and Communication in Kidney and Prostate Cancer, INSERM and University of Strasbourg, School of Medicine, Fédération de Médecine Translationnelle de Strasbourg (FMTS), 67085 Strasbourg, France. ${ }^{9}$ Present address: Department of Urology, Ren Ji Hospital, School of Medicine, Shanghai Jiao Tong University, Shanghai, China. ${ }^{10}$ Present address: INSERM, UMR-1162 "Génomique Fonctionnelle des tumeurs solides", 75010 Paris, France. These authors contributed equally: Clémentine Krucker, Laure Coutos-Thévenot. Correspondence and requests for materials should be addressed to N.R. (email: rochel@igbmc.fr) or to I.B-P. (email: isabelle.bernard-pierrot@curie.fr)
} 
PAR $\gamma$ (peroxisome proliferator-activated receptor gamma) is a transcription factor of the nuclear receptor family that functions predominantly as a heterodimer with RXRa. PPAR $\gamma$ is a key regulator of glucose homeostasis and adipogenesis $^{1,2}$. In addition to its well established role in adipocyte differentiation, it has also been shown to be involved in differentiation in several tissues including the urothelium ${ }^{3}$. Its role in cancer is far less understood. Historically, PPARG was considered to be a tumor suppressor ${ }^{4}$, but several studies showed that it displays pro-tumorigenic effects in neuroblastoma, metastatic prostate cancer, and bladder carcinoma ${ }^{4-8}$.

In bladder cancer, the fourth most frequent cancer in men in industrialized countries, PPARG focal amplifications, resulting in PPAR $\gamma$ and PPAR $\gamma$ target genes overexpression were observed in $12-17 \%$ of the muscle-invasive bladder carcinomas (MIBC) and in $10 \%$ of the non-muscle-invasive bladder carcinomas (NMIBCs) $)^{7,9}$. These copy number alterations of PPARG are associated with luminal tumors, a subtype of bladder tumors accounting for $75 \%$ of non-muscle-invasive bladder carcinomas (NMIBCs) $)^{9,10}$ and $60 \%$ of muscle-invasive bladder carcinomas (MIBCs) ${ }^{11}$. The luminal MIBCs have been shown to display a PPAR $\gamma$ activation signature ${ }^{7,12}$. We previously demonstrated that PPAR $\gamma$ overexpression induces an oncogenic addiction in these tumors by showing that the loss of PPAR $\gamma$ expression inhibited bladder cancer cell viability ${ }^{7}$, most notably observed in cell lines presenting PPARG gain or amplification ${ }^{6,8}$. Recurrent mutations of RXRa (S242F/Y) have also been identified in $5 \%$ of MIBCs and the luminal subgroup of MIBC tumors is enriched in these mutations ${ }^{9,13}$. These $\mathrm{RXR} \alpha$ mutations are gain-of-function mutations that promote ligand-independent activation of PPAR $\gamma$ signaling pathway ${ }^{13,14}$. They drive proliferation of urothelial organoids in a tumor suppressor loss context ${ }^{14}$, render bladder tumor cell growth PPAR $\gamma$-dependent ${ }^{14}$ and promote immune evasion in MIBCs ${ }^{11}$. Hyper-activation of PPAR $\gamma$ signaling, either due to PPARG gene amplification or an RXRa hotspot mutation (S427 F/Y), can be pharmacologically inhibited with PPAR $\gamma$-selective inverse agonists that decrease the viability of PPAR $\gamma$-dependent bladder cancer cells. This highlights PPAR $\gamma$ as a therapeutic target in luminal bladder tumors ${ }^{8}$.

Given this crucial role of the PPAR $\gamma / \mathrm{RXR} a$ pathway in bladder tumors, in this work, we search for other genetic alterations that could drive its activation in both NMIBC and MIBC. By sequencing PPAR $\gamma$ and RXRa in 359 tumors and studying publicly available data for $455 \mathrm{MIBC}$, we identify eight recurrent mutations of PPAR $\gamma$ associated with luminal tumors. Functional analyses reveal that five of these mutations enhance the transcriptional activity of PPAR $\gamma$ and that the activation of PPAR $\gamma$ pathway confers a PPAR-dependence to the cells. Biochemical analyses show that PPAR $\gamma$ mutations favor the recruitment of coregulators. Finally, by a structure-function analysis of three mutations affecting the ligand-binding domain of PPAR $\gamma$, we demonstrate that these mutations promote the adoption of PPAR $\gamma$ active state accounting for the recruitment of coactivators. Our study provides additional genetic evidence for a protumorigenic role of PPAR $\gamma$ in bladder cancer and strengthens the importance of the PPAR $\gamma / \mathrm{RXR} \alpha$ pathway in luminal bladder cancer.

\section{Results}

Recurrent mutations of PPAR $\gamma$ and RXR $\alpha$ in bladder tumors. Using the conventional Sanger's method, we sequenced the PPARG and RXRA exons in 359 bladder tumors (199 of which were NMIBCs) from our CIT series of tumors (carte d'identité des tumeurs) and from a bank of samples collected at Strasbourg hospital, and in 25 bladder cell lines (Supplementary Table 1). We detected PPARG mutations in $3.9 \%$ of the tumors (14/359) and in one cell line, and RXRA mutations in $1.7 \%$ of the tumors $(6 / 338)$, all of which being MIBC, and in one cell line (Fig. 1a and Supplementary Tables 2 and 3). We also analyzed publicly available deep sequencing data from The Cancer Genome Atlas ${ }^{9}$ genomic databases (405 MIBC samples) ${ }^{10}$ (http://cancergenome.nih.gov) and from the Dana Farber \& Memorial Sloan Kettering (MSKCC) cohort $^{15}$ (50 MIBC samples) (Supplementary Table 1). We detected a comparable PPARG mutation rate, $3.1 \%$ of the tumors (14/455), but a higher RXRA mutation rate, $6.1 \%$ of the tumors (28/455) (Fig. 1a and Supplementary Tables 2 and 3) in these tumor series compared to the previous tumor series analyzed. This higher mutation rate was mostly due to the absence of $R X R A$ mutation in NMIBC in the CIT series of tumors. PPARG mutations were not mutually exclusive with either RXRA mutations ( $p$ $=1$ and $p=0.2105$ for conventional and next-generation sequencing, respectively) or PPARG amplification $(p=1$ and $p$ $=1$ for conventional and next-generation sequencing, respectively), as shown by Fisher's exact test analyses. Two mutations (E3K and D7N) were specific to the PPAR $\gamma 2$ isoform (NM_015869) (Supplementary Table 2). We therefore numbered all mutations relative to this isoform, which is 28 amino acids longer than the PPAR $\gamma 1$ isoform (NM_138712) at the N-terminal end. The PPAR $\gamma 2$ isoform is expressed principally in adipocyte cells, as well as in urothelial cells, whereas PPAR $\gamma 1$ is ubiquitously expressed. As we aimed to identify genetic alterations that could activate the PPAR $\gamma /$ RXRa pathway, we focused on recurrent mutations that were likely to be gain-of-function mutations. Of the 21 unique PPAR $\gamma$ mutations identified here in bladder tumors, 6 were recurrent and occurred in both MIBC and NMIBC (Fig. 1b and Supplementary Table 2). Using publicly available data from the COSMIC (http://cancer.sanger.ac.uk/ cosmic) or cBioPortal (http://www.cbioportal.org/) databases, we determined whether the 21 PPAR $\gamma$ mutations identified here were also present in other bladder tumors or in other tumor types. From this analysis, we found a seventh recurrent mutation (E3K) in bladder tumors (Fig. $1 \mathrm{~b}$ and Supplementary Tables 2 and 4). Interestingly, four of these seven recurrent mutations and one unique mutation (M280I) identified in bladder tumors were also reported in other type of tumors in these databases, strengthening their likelihood to be activating mutations (Fig. $1 \mathrm{~b}$ and Supplementary Table 4). We therefore considered in total of eight recurrent mutations of PPAR $\gamma$ for further studies (Fig. 1b, c). These recurrent PPAR $\gamma$ mutations $(p=0.015)$ and $P P A R G$ amplifications $\left(p=1.5 \times 10^{-5}\right)$ were significantly enriched in bladder tumors presenting a PPAR $\gamma$ activation signature, which were mostly luminal tumors $\left(p=2.2 \times 10^{-16}\right)$, as shown by Fisher's exact test. These mutations affect different major functional domains of the PPAR $\gamma$ protein: the N-terminal domain (E3K) including the area around the EGFR phosphorylation sites (S112) (P113S), the DNA-binding domain (R164W, R168K), the ligand-binding domain (S249L, T475M) including the area spatially close to the CDK5 phosphorylation site, S273 [(M280I, I290M)] (Fig. 1b, c). Visualization of recurrent mutations in the three-dimensional (3D) structure of the PPAR $\gamma / \mathrm{RXR} \alpha$ heterodimer revealed that the most frequent recurrent mutation of $\operatorname{PPAR} \gamma(\mathrm{T} 475 \mathrm{M})$ and the hotspot mutation of $R X R \alpha(\mathrm{S} 427 \mathrm{Y} / \mathrm{F})$ affected residues that co-localized spatially at the PPAR $\gamma / \mathrm{RXRa}$ dimer interface, suggesting that both these mutations would affect heterodimer formation (Fig. 1c). Accordingly, RXRa (S427Y/F) mutations have been shown to enhance RXRa interaction with PPAR $\gamma$ and activation of the PPAR $\gamma / R X R a$ pathway ${ }^{13}$.

Recurrent mutations of PPAR $\gamma$ are gain-of-function. We investigated the functional impact of seven of the eight recurrent mutations of PPAR $\gamma$ identified on the transcriptional activity 
a
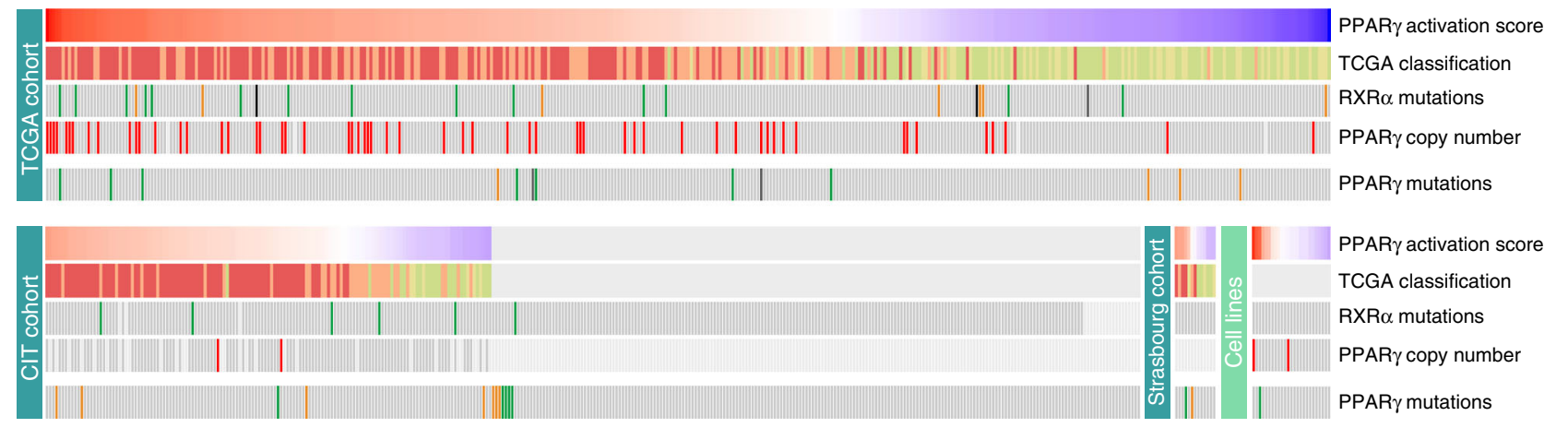

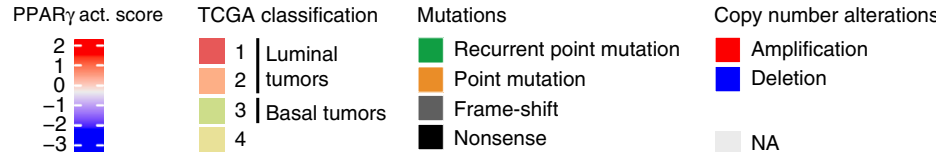

b

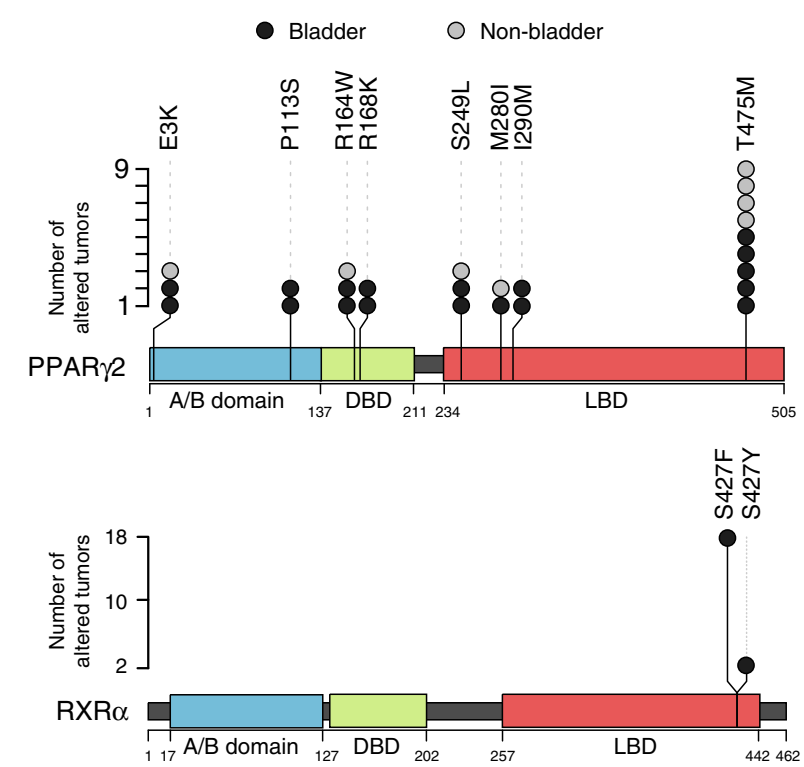

C

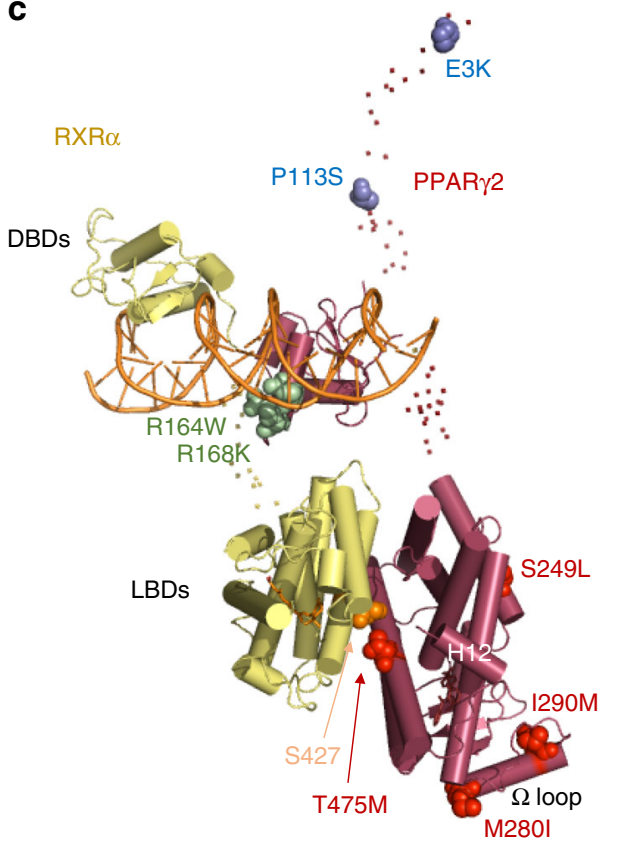

Fig. 1 Mutations of PPAR $\gamma$ and RXR $\alpha$ in bladder tumors. a Oncoprints of PPAR $\gamma$ and $R X R \alpha$ mutations in three series of bladder tumors and a panel of 25 bladder tumor cell lines. Samples were sorted by PPAR $\gamma$ activation score when transcriptomic data were available. Source data are provided as a Source Data file. $\mathbf{b}$ Lolliplot representations of recurrent mutations of PPAR (upper panel) and RXR $\alpha$ (lower panel) identified in the 859 bladder tumors studied here (black circles) or in publicly available data available for other bladder tumors and other types of cancer from the COSMIC and cBioPortal databases (Supplementary Table 4) (gray circles). Sequences are numbered according to the PPAR 2 isoform. A/B: N-terminal domain; DBD: DNA-binding domain; LBD: ligand-binding domain (LBD). c Position of the residues affected by the recurrent PPAR $\gamma$ and RXR $\alpha$ mutations on the full-length PPAR $\gamma$-RXR $\alpha$-DNAcoactivator peptide solution structure ${ }^{51}$. The folded domains are shown in cartoon representation and the disordered hinges and NTDs are shown as dots. The mutated residues are shown as spheres. The residues mutated in PPAR $\gamma$ are colored in blue (A/B domain), green (DBD), and red (LBD). The residue mutated in RXR $\alpha$ is colored in orange. Source data are provided as a Source Data file

of the protein in HEK293FT cells, using a luciferase reporter gene containing three copies of the DR1 sequence of PPAR $\gamma$ DNA response element (PPRE) arranged in tandem and linked to the thymidine kinase promoter $(\mathrm{PPRE}-3 \times-\mathrm{TK})^{16}$. The PPAR $\gamma$ P113S, R168K, S249L, M280I, I290M, and T475M mutant proteins had significantly higher levels of transcriptional activity than the wild type (WT) in the absence of exogenous ligand (two to six times higher), whereas the R164W mutant, which is located in the DNA-binding domain, had levels of activity similar to that of the WT (Fig. 2a). Similar results were obtained in the presence of $1 \mu \mathrm{M}$ rosiglitazone, a synthetic PPAR $\gamma$ ligand (Supplementary Fig. 1A). Consistent with these findings, overexpression of the PPAR $\gamma$ mutants P113S, S249L, M280I, I290M, and T475M, in 5637 cells, in the absence of exogenous PPAR $\gamma$ ligand, significantly enhanced the expression of several known PPAR $y$ target genes (FABP4, ACSL5, and PLIN2) relative to the WT, as shown by RT-qPCR (Fig. 2b and Supplementary Fig. 1B, C). However, overproduction of both the R164W and R168W mutant proteins had an impact on PPAR $y$ target gene expression similar to that of the WT protein (Fig. 2b). Similar results were obtained in presence of $500 \mathrm{nM}$ rosiglitazone (Supplementary Fig. 1D). By combining the results of these two different approaches to measure PPAR $\gamma$ transcriptional activity, we clearly showed that five of the seven recurrent PPAR $\gamma$ mutations analyzed were gain-of-function mutations. The remaining two mutations, R164W and R168K, had no clear apparent 
a

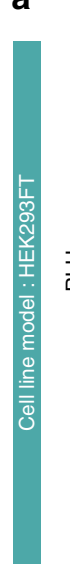

b

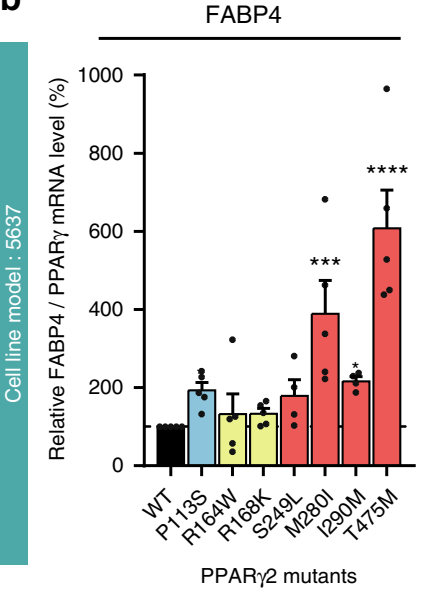

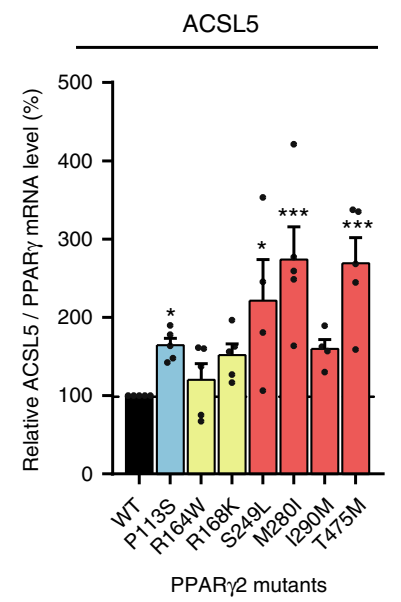

PPAR 2 mutants

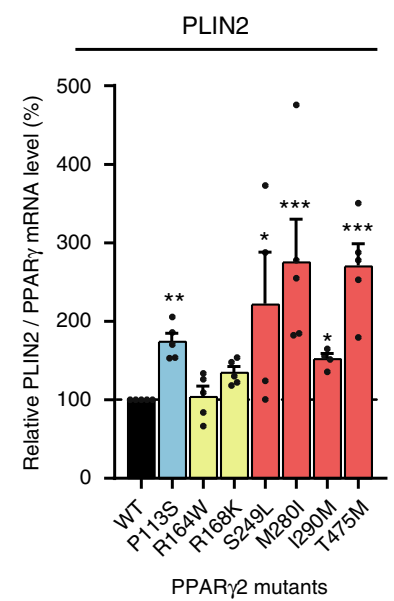

PPAR $\gamma 2$ mutants

A/B domain mutants $\square$ DBD mutants $\square$ LBD mutants
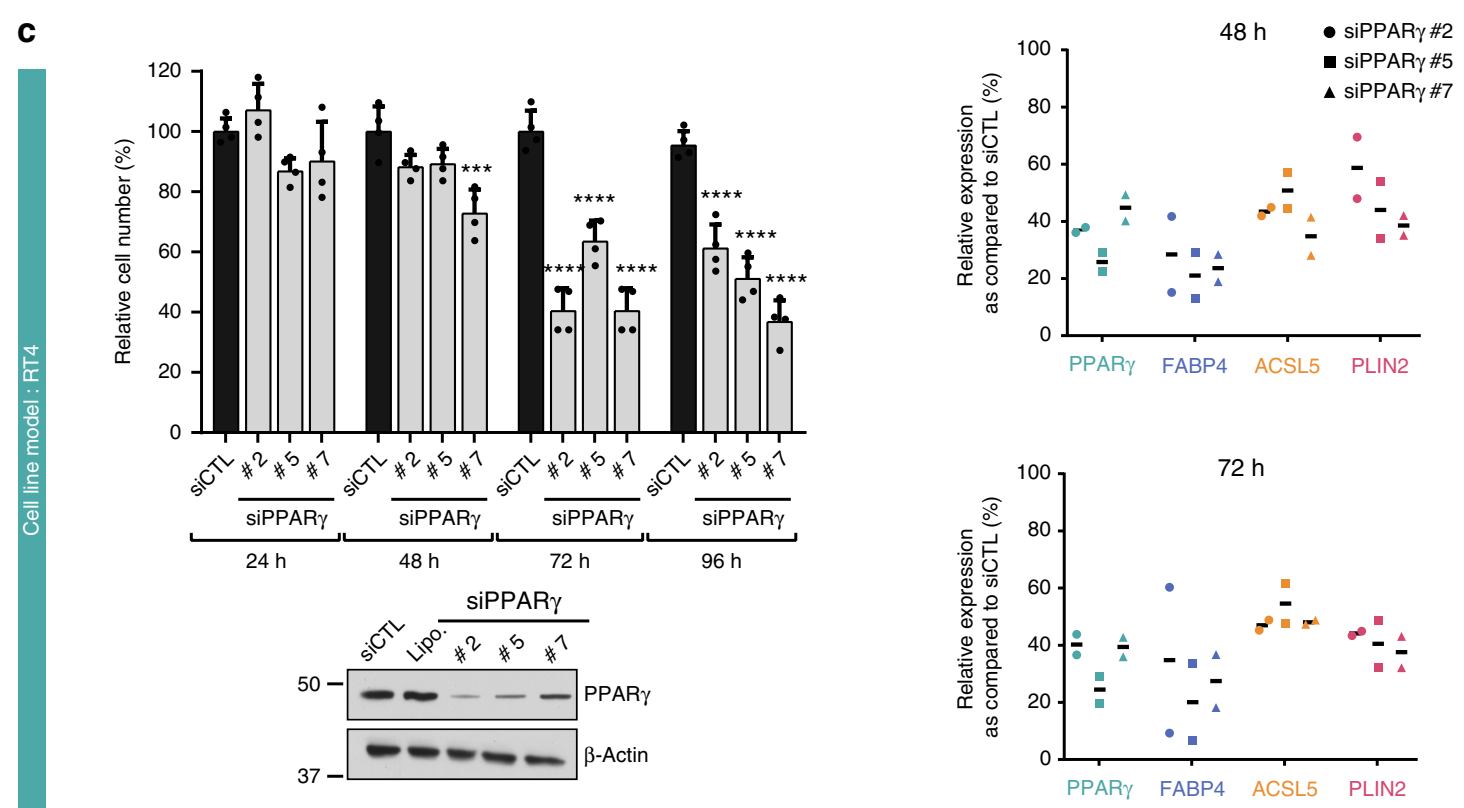

Fig. 2 Transcriptional activity of recurrent PPAR $\gamma$ mutants. a A reporter plasmid containing the firefly luciferase gene under the control of a PPRE-X3-TK promoter was co-expressed in HEK293FT cells with a pcDNA3 vector encoding wild-type (WT) or mutant (P113S, R164W, R168K, S249L, M280I, 1290M, T475M) PPAR 2 . Renilla luciferase, expressed under the control of the CMV promoter, was used to normalize the signal. The data shown are the means \pm SD of one representative experiment conducted in sixtuplate. The results for each mutant were compared with those for the WT in Dunnett's multiple comparisons test, ${ }^{\star} 0.01<p<0.05 ;{ }^{\star \star \star \star} p<0.0001$. b 5637 cells were transiently transfected with a pcDNA3 vector encoding WT or mutant (P113S, R164W, R168K, S249L, M280I, I290M, T475M) PPAR $\gamma$. The expression of all PPAR $\gamma$ forms was checked by western blotting and quantified by RT-qPCR (Supplementary Fig. 1B). The effect of WT PPAR 2 expression on three PPAR $\gamma$ target genes was evaluated by RT-qPCR (Supplementary Fig. 1C). The expression of PPAR $y$ target genes was normalized against PPAR $y$ expression and is expressed as percentage of stimulation relative to the expression induced by WT PPAR $\gamma$. The data are presented as the mean \pm SD of four independent experiments. The results for each mutant were compared with those for the WT in Dunnett's multiple comparisons test: ${ }^{\star} 0.01<p<0.05 ;{ }^{\star \star} 0.001<p<0.01 ;{ }^{\star \star \star} 0.0001<p<0.001$; ${ }^{\star \star \star \star} p<0.0001$. c PPAR $\gamma$ knockdown with three different siRNAs in RT4 cells harboring the PPAR $\gamma$ T475M mutation. PPAR $\gamma$ expression was evaluated by western blotting (lower left panel) $96 \mathrm{~h}$ after transfection, living cells were counted (upper left panel) $24,48,72$, and $96 \mathrm{~h}$ after transfection. Data are presented as means \pm SD of three independent experiments performed in duplicate. The results for each mutant were compared with those for the WT in Dunnett's multiple comparisons test: ${ }^{\star} 0.01<p<0.05 ;{ }^{\star \star} 0.001<p<0.01 ;{ }^{\star \star \star} 0.0001<p<0.001 ;{ }^{\star \star \star \star} p<0.0001$. The expression levels of three PPARG target genes were assessed by RTqPCR for two independent experiment at 48 and $96 \mathrm{~h}$ after transfection (right panel). Data for each experiment are represented. a-d Source data are provided as a Source Data file

effect on PPAR $\gamma$ activity in these two assays. Interestingly, the most frequent mutation, T475M, induced a slightly higher increase in the ligand-independent transcriptional function of PPAR $\gamma$ as compare to any of the recurrent mutations considered (Fig. 2a, b). We used the RT4 cell lines harboring the PPAR $\gamma$-T475M mutation to demonstrate that, like PPARG WT amplification in SD48 and UMUC9 cells ${ }^{7}$ and RXRa
-S427F mutation in HT11978,13 cells, PPAR $\gamma$ mutations render tumor cell growth PPAR $\gamma$-dependent and regulate PPAR $\gamma$ target genes expression. Indeed, PPAR $\gamma$ depletion with siRNAs significantly inhibited, in a time-dependent manner, the growth of RT4 cells (Fig. 2c, left panel) and the expression of several known PPAR $\gamma$ target genes (FABP4, ACSL5, and PLIN2) (Fig. 2c, right panel). 
PPAR $\gamma$ LBD mutations favor its interactions with coregulators. We then used biochemical and biophysical analysis to understand how three mutations located in the ligand-binding domain of PPAR $\gamma$ (M280I, I290M and T475M) promote PPAR $\gamma$ activity. As bacterially expressed PPAR $\gamma$ may contain fatty acids ${ }^{17}$, the purified PPAR $\gamma$ LBD WT and mutants (Supplementary Figs. 2 and 3) were analyzed by native electrospray mass spectrometry (Supplementary Fig. 4). The results indicated the absence of any bound ligands. The addition of the potent full PPAR $\gamma$ agonist, GW1929, an $\mathrm{N}$-aryl tyrosine derivative ${ }^{18}$ and coactivator peptide led to the formation of ternary complexes for all constructs (Supplementary Fig. 4, right panel). We characterized the interaction between the ligand-binding domains of the PPAR $\gamma$ WT and mutants and the WT RXRa monomer by analytical ultracentrifugation (Supplementary Fig. 5A), which showed that as for the WT, all mutant proteins were able to form heterodimer with
RXRa. To further quantify the interaction between monomeric PPAR $\gamma$ and RXRa ligand-binding domains, we used surface plasmon resonance (SPR) (Fig. 3a and Supplementary Fig. 5B, C). PPAR $\gamma$ T475M, localized at the dimer interface, exhibits an increase by 2 -fold of the binding affinity to RXR $\alpha$ compared to PPAR $\gamma$ WT (Fig. 3a), as a consequence of a slower dissociation rate of the dimer (Supplementary Fig. 5B, C). In RXRa the S427F mutation is also spatially localized at the PPAR $\gamma / \mathrm{RXR} \alpha$ dimer interface, and it has been shown to similarly stabilize the heterodimer ${ }^{13}$. In contrast, PPAR $\gamma$ M280I shows similar affinity for RXRa as PPAR $\gamma$ WT.

The functional profile of $\operatorname{PAAR} \gamma$, analogous to many other nuclear receptors, is determined by the selective use of transcriptional coregulators that ultimately control the transcriptional output of the target genes. The recruitment of coactivators by PPAR $\gamma$ is primarily determined by the interaction of

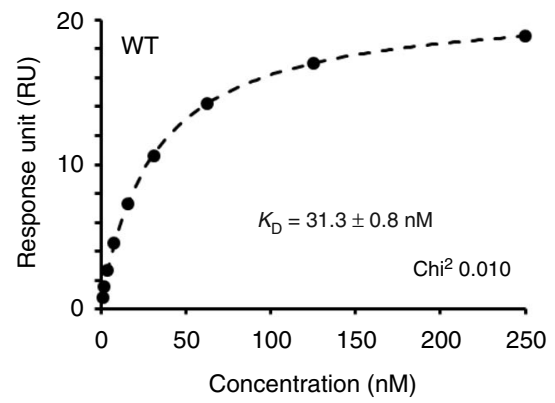

b
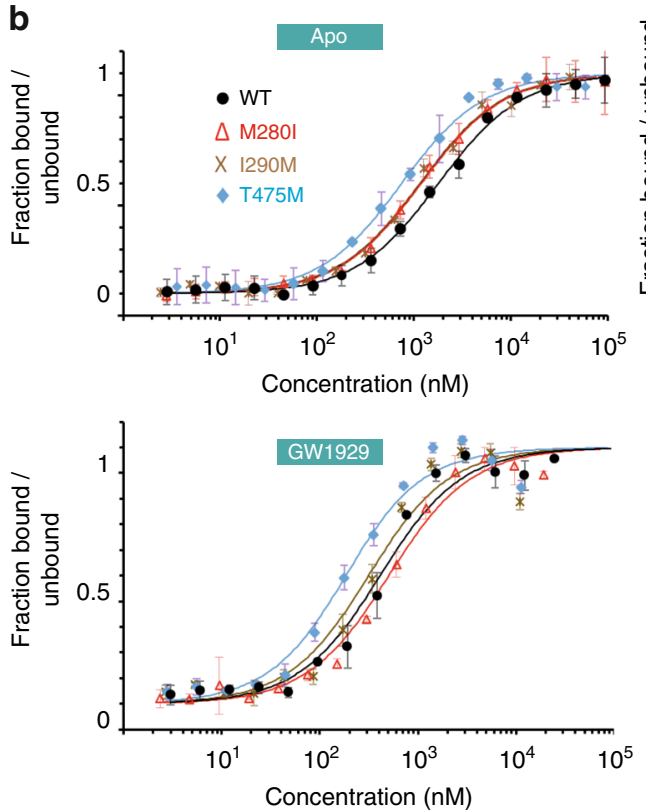
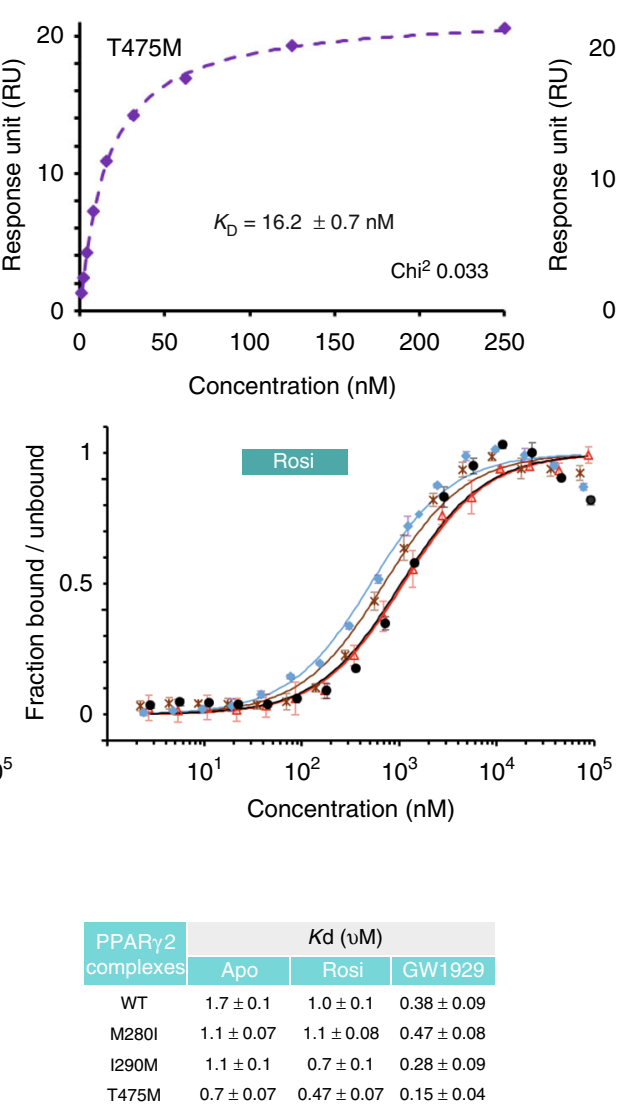
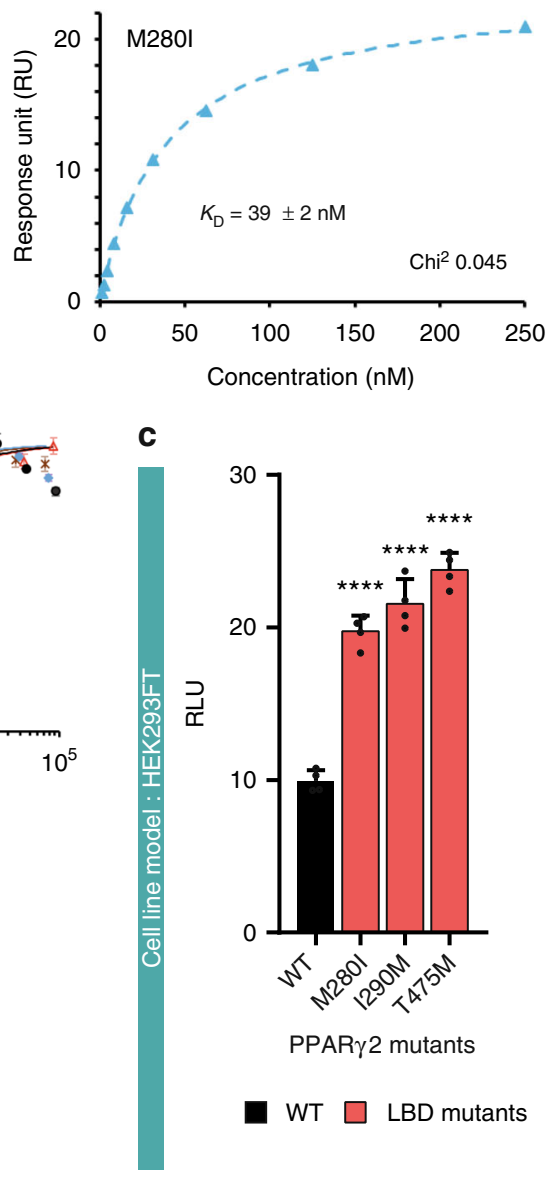

Fig. 3 Effect of the PPAR $\gamma$ mutations on RXR $\alpha$ and coactivator interactions. a Representative data set used for SPR (surface plasmon resonance) analysis of the interactions of PPAR $\gamma$ WT or mutants with RXR $\alpha$ showing enhanced interaction between PPAR $\gamma$ T475M and RXR $\alpha$. Report point $4 \mathrm{~s}$ before injection stop was used for the analysis. Equilibrium responses are plotted as a function of total protein concentration and fit to simple 1:1 binding isotherms. Data analysis by 1:1 kinetic model and mean kinetic parameters and equilibrium dissociation constants are reported in Supplementary Fig. 4. b Effect of PPAR $\gamma$ mutations on the PGC1 $\alpha$ peptide interaction. The binding affinity of the PGC1 $\alpha$ NR1 motif for the purified WT and mutant PPAR $\gamma$ LBDs, as determined by microscale thermophoresis. Unlabeled PPAR $\gamma$ LBD protein was titrated into a fixed concentration of fluorescently labeled PGC1 $\alpha$ peptide in the absence of ligand (top left), in the presence of three equivalents of rosiglitazone (top right) or of three equivalents of GW1929 (bottom left). Isotherms averaged over three consecutive measurements and fitted according to the law of mass action to yield the apparent $K_{d}$. Each plot is representative of at least two independent experiments performed with different batches of protein preparation. c Mammalian two-hybrid analysis reveals increased interaction of PPAR $\gamma$ mutants (M280I, I290M and T475M) with MED1 coactivator domain. pG5-Firefly luciferase reporter plasmid was co-expressed with VP16-PPARG (WT or mutants) and with GAL4-DNA-binding domain-fused MED1. Renilla luciferase, expressed under the control of the CMV promoter, was used to normalize the signal. The data shown are the means \pm SD of one representative experiment conducted in quadruplicate. Results (means \pm SD) of three independent experiments are also provided as Supplementary Fig. 16. The results for each mutant were compared with those for the WT in Dunnett's multiple comparisons test, ${ }^{\star} 0.01<p<0.05 ;{ }^{\star \star} 0.001<p<0.01$. a-c Source data are provided as a Source Data file 
coactivator LXXLL motifs with the receptor LBD. To analyze the functional consequences of the mutations in the LBD, coactivator peptide recruitment by the LBD was monitored. We measured the interaction between monomeric WT or mutant forms of PPAR $\gamma$ and a fluorescently labeled coactivator peptide of PGC1 $\alpha$ (PPARGC1A), by MicroScale Thermophoresis. In the absence of ligand, the three mutations enhanced the interaction with coactivator peptide compared to PPAR $\gamma$ WT (Fig. 3b). The addition of a full agonist, rosiglitazone (Fig. 3b), enhanced the interaction between PPAR $\gamma$ and PGC1a coactivator peptide for PPAR $\gamma$ I290M and T475M, but not for PPAR $\gamma$ M280I. The addition of another potent full agonist of PPAR $\gamma$, GW1929, enhanced yet again the interaction for all mutants, as well as the WT (Fig. 3b). Consistent with the functional data (Fig. 2a, b), monomeric PPAR $\gamma$ T475M had the highest affinity for the coactivator peptides (Fig. 3b). This strongest structural stabilizing effect of T475M mutant on PPAR $\gamma$-coactivator peptide complex was also observed by ion mobility mass spectrometry which showed significantly improved gas phase stability in collision induced experiments of T475M compared to WT and other PPAR $\gamma$ mutants (Supplementary Figs. 6 and 7). Mammalian two-hybrid assay in HEK293FT cells using VP16-fused PPAR $\gamma$
(WT, M280I, I290M, and T475M), GAL4-DNA-binding domainfused coactivator MED1 and pG5-LUC reporter confirmed that in the context of full protein, the three mutations enhanced interaction with MED1 coactivator domain compared to PPAR $\gamma$ WT (Fig. 3c). Together, these data suggest that the three mutations considered, M280I, I290M and T475M, promote the adoption of an agonist conformation by PPAR $\gamma$ in the absence of ligand, thereby enhancing coactivator interaction.

PPAR $\gamma$ LBD mutations stabilize an active conformation. To gain structural insight into the mechanism responsible for the increases in activity and coactivator interaction, we analyzed the structures of the PPAR $\gamma$ LBD T475M, M280I and I290M mutants (Supplementary Table 5). The PPAR $\gamma$ T475M LBD mutant was crystallized in its apo form and in complex with GW1929 and the PGC1a coactivator peptide (Fig. 4a and Supplementary Fig. 8). In both functional states, PPAR $\gamma$ T475M crystallized as a homodimer. More than 150 crystal structures of the PPAR $\gamma$ LBD have been deposited in the Protein Data Bank (www.rcsb.org) ${ }^{19}$, and in many of them, the PPAR $\gamma$ LBD crystallized as a homodimer displaying the canonical dimer interface observed in the heterodimer complex. Of these structures, the apo protein and some
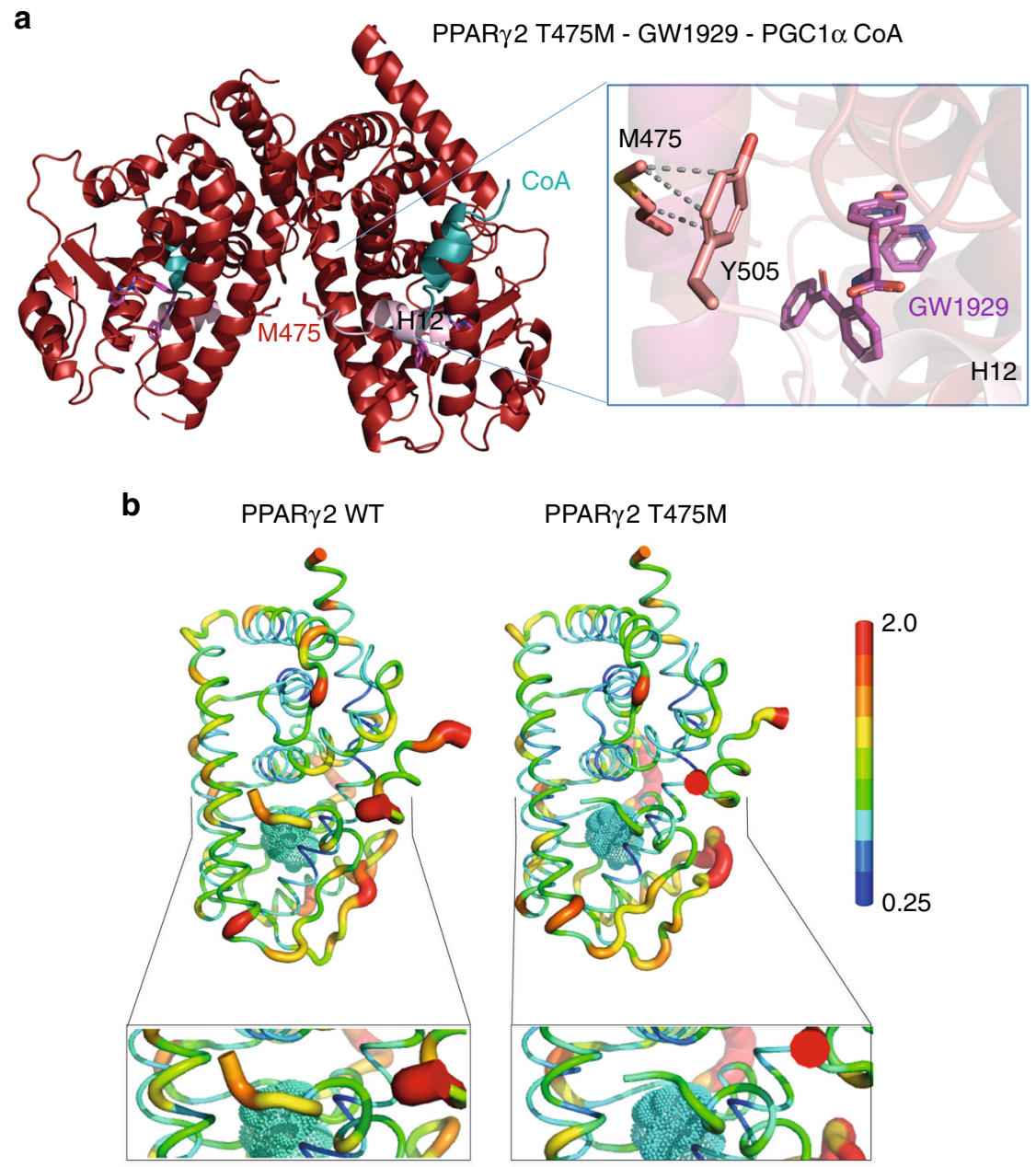

Fig. 4 Structural properties of PPAR $\gamma$ T475M. a Crystal structure of the PPAR $\gamma$ T475M LBD (red) in complex with GW1929 (violet) and the PGC1 $\alpha$ coactivator peptide (blue). The C-terminal H12 helices are shown in light pink. Overall fold of the homodimer complex, showing the T475M mutations at the dimer interface as a stick representation. Right: Close-up of the regions around the mutation, showing its interactions with the terminal Y505 residue responsible for stabilizing the agonist conformation. b The PPAR $7475 \mathrm{M}$ mutation modulates the structural dynamics of the LDB. Mean structure and atomic fluctuations of the holo PPAR $\gamma$ WT LBD-coactivator (left) and holo PPAR $\gamma$ T475M LBD-coactivator (right) complexes, with the rosiglitazone ligand (in cyan), and the scale of flexibility shown. Bottom: Close-up of the C-terminal H12 helix of the WT and mutant LBDs, as determined from molecular dynamics simulations 
complexes with partial agonists display one monomer in an active conformation and one monomer in an inactive conformation with a different positioning of helix $12^{20,21}$. Helix 12 is key regulatory structural element in the activation function 2 interacting with coregulators. These 2 conformations are generally designated as fully active and inactive ${ }^{21}$. Although these helix 12 conformations observed in the crystal structures are influenced to some degree by crystal packing, they reflect the dynamic character of helix 12 in absence or in presence of a partial agonist ligand. This was further confirmed by solution structural methods $^{22}$. Interestingly, in contrast to the structures of WT PPAR $\gamma$ in its apo form, PPAR $\gamma$ T475M apo displays an agonist conformation in both homodimer LBDs, indicating a stabilization of helix 12 in an active conformation even in absence of any agonist ligand (Supplementary Fig. 8).

In a similar way, the holo PPAR $\gamma$ T475M-GW19129-PGC1a ternary complex crystallized as a homodimer (Fig. 4a) and the structure agrees with the crystal structure of another $\mathrm{N}$-aryl tyrosine derivative complex, GI26257023. The GW1929 ligand binds in a U-shaped conformation with the carboxyl group of GW19219 making hydrogen bonds with S317, H351, H477, and Y501 (Supplementary Fig. 9). The pyridinyl tail is directed towards the $\beta$-sheet and its nitrogen atom makes an $\mathrm{H}$-bond with a bound water molecule. The benzophenone attached to the $\mathrm{N}$-aryl tyrosine, also present in GI262570, forms additional hydrophobic interactions, not available to rosiglitazone, explaining their increased PPAR $\gamma$ binding affinity and enhanced coactivator interaction ${ }^{18,22,24}$. The mutated residue $\mathrm{T} 475 \mathrm{M}$ interacts with the C-terminal Y505 (Fig. 4a) stabilizing helix 12 into the active conformation and leading to a more stable interaction with the coactivator peptide. Interestingly, a similar interaction stabilizing PPAR $\gamma$ in the active agonist conformation was observed in the crystal structure of a heterodimer complex of holo WT PPAR $\gamma$ and the RXRa S427F mutant ${ }^{13}$. A similar observation was made by molecular dynamics (MD) simulations of the $\mathrm{WT}^{20}$ and T475M PPAR $\gamma$ LBD (Fig. 4b). Starting from a previously determined crystal structure of the WT protein ${ }^{20}$, the point mutation was modeled into the WT structure. Separate 100 ns simulations of monomeric WT and T475M mutant PPAR $\gamma$ LBDs in both apo form and in complex with rosiglitazone were performed. We found that the T475M mutation decreased the structural flexibility of the protein, particularly that of the Cterminal H12 helix (Fig. 4b), through direct interaction between the M475 and the C-terminal Y505, as observed in the structure of PPAR $\gamma$ T475M in complex with GW1929. In the WT structure, the analogous interaction (between T475 and Y505) does not appear; the M475-Y505 interaction formed during the molecular dynamics simulations (Supplementary Fig. 10). The decrease in the flexibility of the C-terminal H12 helix in the T475M mutant led to a lower flexibility of the coactivator peptide, resulting in a more stable complex. Free energies of coactivator interaction were calculated by the MM/GBSA ${ }^{25}$ method on 100 structures taken from the MD simulations at regular intervals. This analysis yielded binding energy estimates of $-43 \pm 7 \mathrm{kcal} / \mathrm{mol}$ and $-48 \pm 5 \mathrm{kcal} / \mathrm{mol}$ for WT PPAR $\gamma$ and for the T475M mutant, respectively, consistent with the trends observed in the experimental data of coactivator interaction. In this application, the MM/GBSA binding energies are used to rank interactions and not to calculate absolute binding-free energies.

As the T475M mutation is located at the dimer interface, we modeled the structure of the heterodimer formed by PPAR $\gamma$ T475M (Supplementary Fig. 11). The results suggest that T475M will strengthen the heterodimer through interactions with L430 and S427 in contrast to WT, in agreement with the experimental data. Molecular dynamics simulations of a PPAR $\gamma$-T475M/RXRa heterodimer model showed that the heterodimer is more structurally stable than the WT and, as observed in the monomer simulations, the coactivator peptide displayed reduced flexibility (Supplementary Fig. 11C). Thus, our structural analysis of PPAR $\gamma$ T475M indicates that the mutation stabilizes PPAR $\gamma$ helix 12 in the active conformation as well as the heterodimer with RXRa, leading to a stronger binding to coactivators. This mechanism may explain PPAR $\gamma$-dependent transcription program activation.

The two other mutants of interest here are localized in the $\Omega$ loop of the PPAR $\gamma$ LBD; M280I is in the helix $2^{\prime}$ of the $\Omega$ loop and $\mathrm{I} 290 \mathrm{M}$ is in the loop between helix $2^{\prime}$ and helix 3 . The $\Omega$ loop is often poorly defined in the electron density maps of the published structures of PPAR $\gamma$, adopting varying conformations depending on the nature of the ligand. It is thought to serve as a gate to the ligand-binding pocket ${ }^{26}$ and also serve as an alternative ligand-binding site for some ligands ${ }^{27,28}$. The PPAR $\gamma$ M280I LBD crystallized in complex with GW1929 and PGC1a peptide as a homodimer (Fig. 5a) and PPAR $\gamma$ I290M in complex with GW1929 as a monomer (Supplementary Fig. 12B). The conformation and interactions of GW1929 in the PPAR $\gamma$ LBPs is similar in all complexes (Supplementary Fig. 9A). While helix $2^{\prime}$ is similarly positioned in all complexes, the flexible loop connecting helix $2^{\prime}$ and helix 3 shows different conformations and is partially resolved in some complexes. The M280I mutant stabilizes helix 3, notably through specific interactions with V305 (Fig. 5a), that, in turn stabilizes PPAR $\gamma$ in the agonist conformation. Recall that the coactivator interaction is via the activation function 2 coregulator surface composed of helix 3, 4, 5, and helix $12^{20}$. For the I290M mutant, the surrounding loop connecting helix $2^{\prime}$ and helix 3 shows some flexibility as indicated by the poor density of some side chains residues and higher temperature factors. However, the structural analysis of I290M-GW1929 complex indicates that I290M interacts more strongly with I369, which contacts the ligand (Supplementary Fig. 12B) and may stabilize the $\beta$-sheet, as well as helix 3. Following the protocol used in the previously described simulations, molecular dynamics simulations of the M280I and I290M LBDs were carried out. From the average structure calculated over the last $10 \mathrm{~ns}$ of each simulation, we found interactions that stabilized the $\Omega$ loop region via helix 3 and further stabilization of $\mathrm{H} 12$ activation function 2 for both the M280I and the I290M mutants. In the case of M280I mutant, I280 formed a stable interaction with V305 of H3, the N-terminal end of H3. This interaction was also seen in the crystal structure of the M280I mutant described above. In the I290M variant, M290 forms an interaction with Phe315 of H3, which interacts with His494 at the N-terminal end of H12. Stabilization of H12 leads to a stabilization of the coactivator peptide, as indicated by the decreased atomic fluctuations shown in Fig. 5b, which shows the rms fluctuations mapped onto the $3 \mathrm{D}$ surface of the proteins complexes. The free energies of coactivator binding to M280I and I290M were also estimated by the MM/GBSA method on structures taken from the MD simulations at regular intervals. The binding energies, $-40 \pm 7 \mathrm{kcal} / \mathrm{mol}$ and $-45 \pm 7 \mathrm{kcal} / \mathrm{mol}$ for M280I and I290M, respectively, are consistent with the trends observed in the experimental data of coactivator interaction. In particular, the mutant I290M shows an enhanced binding free energy with respect to the WT.

We used hydrogen/deuterium exchange coupled to mass spectrometry (HDX-MS) to investigate complementary structural information about the effect of T475M and M280I mutations on PPAR $\gamma$ structure. Of note, due to lack of reproducibility in HDX-MS data acquisition on I290M, these data were excluded from the present study. HDX-MS is a powerful readout to monitor protein conformational dynamics at peptide resolution, by monitoring the $\mathrm{D}$ exchange of amide hydrogens with deuterated solvent ${ }^{24}$. In order to evidence PPAR $\gamma$ point mutation 

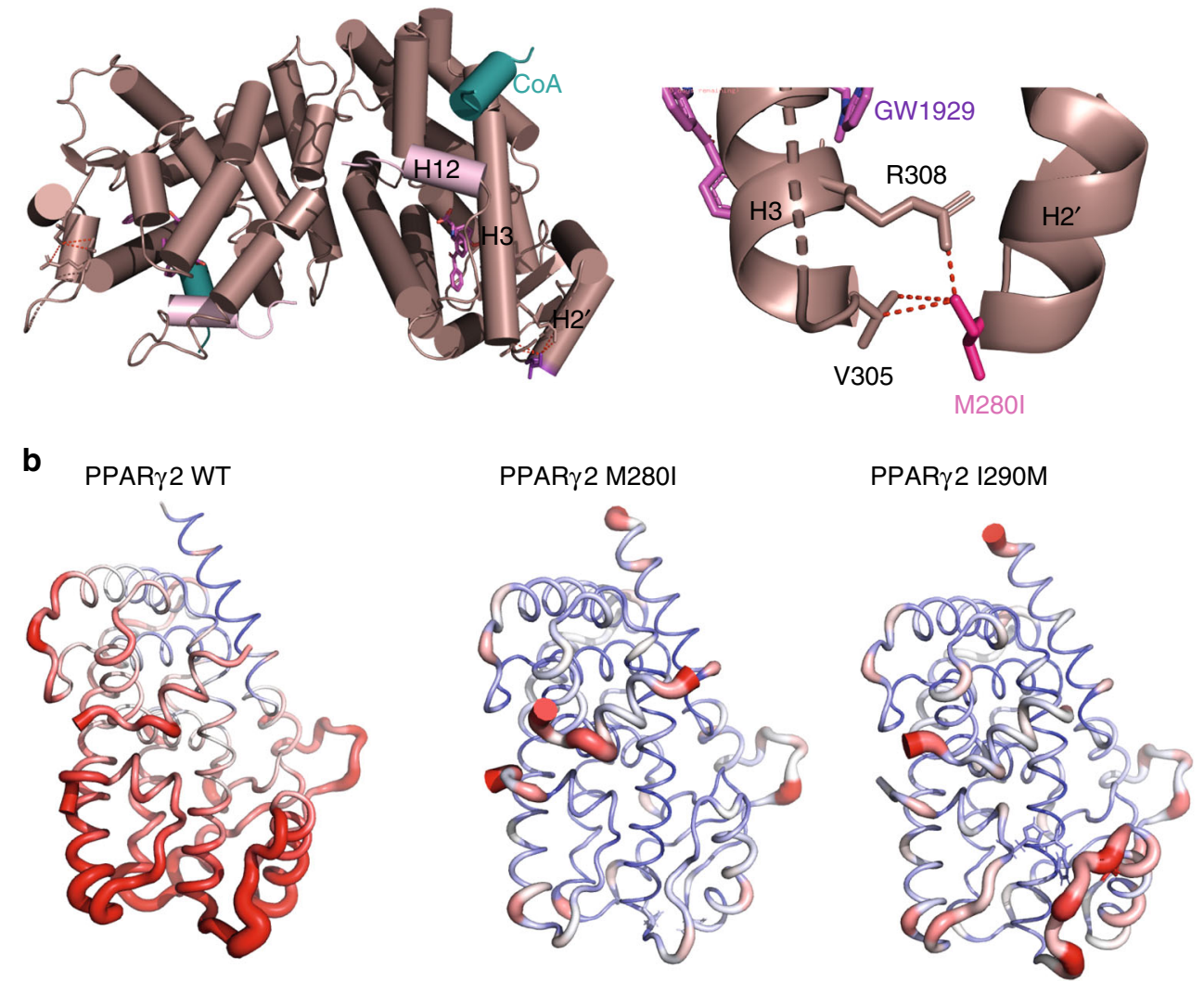

Fig. 5 Impact of PPAR 1 M280I and I290M mutations on the structure and dynamics. a Crystal structure of the PPAR $\gamma$ M280I LBD (plum) in complex with GW1929 (light red) and the PGC1 $\alpha$ coactivator peptide (blue). The C-terminal H12 are shown in light pink. Right: Close-up of the regions around the mutation, showing its interactions stabilizing helix 3. b The atomic fluctuations calculated from the molecular dynamics simulation (last 10 ns) of the holo form of PPAR $\gamma$ complexes with rosiglitazone and coactivator peptide. The fluctuations are mapped onto the 3D surface of the proteins complexes. The color scale goes from blue (less flexible) to red (most flexible) between 0 and $2.5 \AA$. The thickness of the tube also reflects the flexibility (thicker corresponds to more flexible). Left: WT; middle: M280I; right: I290M

effects on secondary structures of PPAR $\gamma /$ PGC1a complexes, we performed pairwise comparison of deuterium uptakes of PPAR $\gamma$ WT/PGCla to PPAR $\gamma$ mutant/PGC1a (Supplementary Fig. 13). For this, relative fractional uptakes (RFU) of PPAR $\gamma$ mutants/ PGC1a were subtracted from the PPAR $\gamma$ WT/PGC1a RFU and the significance of the RFU difference plots was validated using the MEMHDX software ${ }^{29}$ ( $p<0.01$, Supplementary Fig. 13). Overall, PPAR $\gamma$ mutant proteins presented a lower level of deuterium uptake compared to WT when bound to PGC1 $\alpha$, highlighting the fact that PPAR $\gamma$ mutant/PGC1a complexes present several regions less flexible or less exposed to solvent than PPAR $\gamma$ WT/PGCla complex (Supplementary Fig. 14).

Comparison of PPAR $\gamma$ T475M/PGC1a to PPAR $\gamma$ WT/PGC1a RFUs revealed that different regions of the T475M mutant presented a significant lower deuterium uptake (Supplementary Fig. 14, upper panel), including $\mathrm{H} 2, \mathrm{H} 2$ ', N-terminal H3, H6, Nterminal $\mathrm{H} 8$, helix $\mathrm{H} 10$, and the $\mathrm{H} 12$ helix. These regions are thus more protected from deuterium exchange for PPAR $\gamma$ T475M compared to PPAR $\gamma$ WT, which might be in favor of a decreased conformational flexibility of PPAR $\gamma$ T475M compared to PPAR $\gamma$ $\mathrm{WT}$, in agreement with MD simulation data.

For PPAR $\gamma$ M280I a significantly different behavior was observed compared to PPAR $\gamma$ T475M (Supplementary Fig. 14, middle panel). Decreased deuterium uptake was observed around the mutation site (from the C-terminal H1 towards the mutation site and until $\mathrm{H} 3$, including the $\Omega$ loop). In addition, the $\beta$-sheet region, $\mathrm{H} 6$ helix, the loop between $\mathrm{H} 8$ and $\mathrm{H} 9$ and finally the region encompassing $\mathrm{H} 10, \mathrm{H} 11$, and $\mathrm{H} 12$ helices showed also significant decrease in deuterium uptake, which might correspond to a decreased conformational flexibility. Our HDX-MS data for the PPAR $\gamma$ M280I are consistent with MD simulations highlighting a stabilization of the $\Omega$ loop, $\mathrm{H} 3$ and $\mathrm{H} 12$ helices in the M280I mutant protein, compared to WT. Altogether, HDX-MS data are thus in agreement with our structural analysis of the structural elements involved in agonist conformation stabilization of the T475M and M280I mutant proteins.

\section{Discussion}

Taken together, our study highlighted the existence of recurrent driver gain-of-function PPAR $\gamma$ mutations in a particular type of cancer: luminal bladder tumors. Frequent PAX8-PPAR $\gamma$ fusions have already been reported in follicular thyroid tumors $(30 \%$ of these tumors), but these proteins, which also have a protooncogenic role, are likely to act in a dominant negative manner ${ }^{30}$. The P113S mutation enhanced PPAR $\gamma$ activity, probably by inhibiting S112 phosphorylation by MAP kinases, as shown for the only gain-of-function mutation of PPAR $\gamma$ reported to date, P113G, which is associated with obesity ${ }^{31}$. We have also identified two recurrent mutations affecting the DNA-binding domain of $\operatorname{PPAR} \gamma$, but the functional impact of these mutations was not as clear as for the other mutants and requires further investigation. However, we clearly showed that the three recurrent mutations affecting the ligand-binding domain of PPAR $\gamma$ altered the 
conformation and structural dynamics of the protein, stabilizing helix $\mathrm{H} 12$ and so promoting the adoption of the active form by the receptor even in the absence of ligand. These mutations also favor the recruitment of coactivators. Such effects have already been described for mutations affecting the ligand-binding domain of another hormone receptor, ER, in breast cancer, these mutations however being found exclusively in hormone-resistant metastases, not in primary tumors ${ }^{32}$. Mutations affecting the ligand-binding domain of the androgen receptor have also been observed in metastatic or castration-resistant prostate cancers and these mutations enable the protein to bind other ligands ${ }^{33}$. Our demonstration that the activation of PPAR $\gamma$ by point mutation, like PPARG amplification ${ }^{7}$ or RXRa mutation ${ }^{8,13}$, confers bladder cancer cells a PPAR $\gamma$ dependency, provides additional genetic evidence for a pro-tumorigenic role of PPAR $\gamma$ in bladder cancer and strengthens the importance of the PPAR $\gamma / R X R \alpha$ pathway in luminal bladder cancer. RXRa hotspot mutations appear to be specific to bladder cancer. By contrast, the PPAR $\gamma$ gain-offunction mutations identified here are also observed in other types of cancer. In particular, T475M mutations are observed in four different types of cancer (Supplementary Table 4). Moreover, other recurrent mutations not identified in bladder cancer have been observed in different types of cancer, including melanoma and prostate carcinoma (Supplementary Table 6), and these mutations may also likely be activating mutations. The activation of PPAR $\gamma$ by mutations may, therefore, result in PPAR $\gamma$ dependence not only in bladder cancer, but also in other types of cancer, reinforcing the importance of developing new pharmacological approaches targeting the ligand-independent activity of PPAR $\gamma$ in tumors. Such treatments would also favor the immune response in bladder tumors ${ }^{13}$. A better understanding of the molecular basis of the pro-oncogenic activity of PPAR $\gamma$ in bladder luminal tumors might also make it possible to propose alternative therapeutic options for targeting this pathway without directly inhibiting PPAR $\gamma$, which could lead to diabetes or lipodystrophy ${ }^{34}$.

\section{Methods}

Human bladder samples. We used DNA extracted from 359 bladder tumors. The flash-frozen tumor samples were stored at $-80^{\circ} \mathrm{C}$ immediately after transurethral resection or cystectomy. All tumor samples contained more than $80 \%$ tumor cells, as assessed by the hematoxylin and eosin (H\&E) staining of histological sections adjacent to the samples used for DNA extraction. All subjects provided informed consent and the study was approved by the institutional review boards of the Henri Mondor, Foch, Institut Gustave Roussy and Strasbourg Hospitals.

Sanger sequencing. The coding exons and splice junctions of PPARG and RXRA were amplified from genomic DNA by PCR with gene-specific primers available on request and sequenced by the Sanger method. For tumors, the somatic status of the identified mutation was confirmed by sequencing normal DNA from blood. Sequencing was performed for 25 bladder cell lines: 5637, BFTC-905, CAL29, EJ138, HT1376, J82, JMSU1, KK47, L1207, MGHU3, RT112, RT4, SCaBER, SD48, SW1710, T24, TCCSup, UMUC1, UMUC5, UMUC6, UMUC9, UMUC10, UMUC16, VMCUB1, and VMCUB3. The identity of the cell lines used was checked by analyzing genomic alterations with comparative genomic hybridization arrays (CGH array), and FGFR3 and TP53 mutations were checked with the SNaPshot technique (for FGFR3) or by classical sequencing (for TP53). The results obtained were compared with the initial description of the cells. We routinely checked for mycoplasma contamination.

Calculation of a PPAR $\boldsymbol{\gamma}$ activation score. Based on our previously described PPAR $\gamma$ activation signature encompassing 148 genes $^{7}$, we defined a subset of 77 genes that were also significantly more expressed in the $25 \%$ of tumors with the strongest PPAR $\gamma$ expression than in the $25 \%$ of tumors with the lowest levels of PPAR $\gamma$ expression in TCGA datasets $(N=405 \text { tumors })^{10}$. The PPAR $\gamma$ activation score is the mean of the centered expressions of these 77 genes for each tumor.

Materials and chemicals. Rosiglitazone and GW1929 were purchased from Tocris Bioscience. The fluorescent PGC1 $\alpha$ peptide (137-EAEEPSLLKKLLLAPA-152) was purchased from Thermo-Fisher. The PGC1 $\alpha$ peptide (139-EEPSLLKKLLLAPA-152) was synthesized by Pascal Eberling (IGBMC peptide synthesis common facility).
Plasmid constructs. The pcDNA3-PPAR $\gamma 2$ and PPRE-X3-TK-luc were generously provided by Pr. Chatterjee (Institute of Metabolic Science, IMS, Cambridge) and Bruce Spiegelman (Addgene plasmid \#1015), respectively. We used pcDNA3.1-PPAR $\gamma 2$ and the QuikChange II Site-Directed Mutagenesis Kit (Agilent Technologies) according to the manufacturer's protocol, to generate all the mutations. Mutations were confirmed by DNA sequencing. The GAL4-DNAbinding domain cloning vector $\mathrm{pM}$ and the activation-domain cloning vector pVP16 are part of the Mammalian Matchmaker Two-Hybrid Assay kit (BD Biosciences Clontech). The construct pM-MED1 (510-787) expressing the Gal4 DBD-MED1 nuclear receptor interacting domain was provided by Lieve Verlinden (KU Leuven, Belgium) ${ }^{35}$.

Cell culture and transfection. The HEK293FT human cell line and the RT4 and 5637 human bladder tumor-derived cell lines were obtained from DSMZ (Heidelberg, Germany). HEK293FT cells were cultured in DMEM, whereas 5637 and RT4 cells were cultured in RPMI. Media were supplemented with $10 \%$ fetal calf serum (FCS). Cells were incubated at $37^{\circ} \mathrm{C}$, under an atmosphere containing $5 \% \mathrm{CO}_{2}$.

For reporter gene assays, HEK293FT cells were plated in 96-well plates $(30,000$ cells per well) and transfected with $30 \mathrm{ng}$ pcDNA3-PPAR 2 (WT or mutated), $50 \mathrm{ng}$ PPRE-X3-TK-luc and $6 \mathrm{ng}$ pRL-SV40 (Promega), in the presence of the Fugene HD transfection reagent (Promega), in accordance with the manufacturer's protocol. Luciferase activity was determined $48 \mathrm{~h}$ later, with the Dual-Glo ${ }^{\circledR}$ Luciferase Assay System (Promega), according to the manufacturer's instructions, and the results obtained were normalized with the Renilla luciferase signal obtained with the pRL-SV40 plasmid.

For PPAR $\gamma 2$ overexpression in the 5637 cell line, we used six-well plates, 250,000 cells seeded per well. These cells were transfected $24 \mathrm{~h}$ later with $2.5 \mu \mathrm{g}$ of pcDNA3-PPAR $\gamma 2$ (WT or mutated) in the presence of the Fugene HD transfection reagent (Promega). RNAs were extracted with the RNA easy mini kit (Qiagen) and proteins were extracted by cell lysis in Laemmli buffer (50 mM Tris- $\mathrm{HCl}$ (pH 7.5), $250 \mathrm{mM} \mathrm{NaCl}, 1 \%$ SDS) supplemented with protease inhibitors and phosphatase inhibitors (Roche) $48 \mathrm{~h}$ after transfection.

For transfection with siRNA, RT4 cells were used to seed six-well plates at a density of 300,000 cells/well. Cells were transfected with $20 \mathrm{nM}$ siRNA in the presence of Lipofectamine RNAi Max reagent (Invitrogen), in accordance with the manufacturer's protocol. siRNAs were purchased from Qiagen. For the control siRNA, we used a control targeting luciferase (SI03650353). We used three PPAR $\gamma$ siRNAs designed to knockdown the expression of all known mRNA isoforms. The sense-strand sequences were: PPARG siRNA\#2:

GACAAAUCACCAUUCGUUATT, PPARG SiRNA\#5:

GCGACUUGGCAAUAUUUAUTT and PPARG siRNA\#7:

CGGAGAACAAUCAGAUUGATT.

Cells were detached from the plates with trypsin $96 \mathrm{~h}$ after transfection, counted using a hemocytometer and lysed in Laemmli buffer $(50 \mathrm{mM}$ Tris- $\mathrm{HCl}(\mathrm{pH} 7.5)$, $250 \mathrm{mM} \mathrm{NaCl}, 1 \%$ SDS) supplemented with protease inhibitors and phosphatase inhibitors (Roche)

For mammalian two-hybrid assay, HEK293FT cells were plated in 96-well plate (30,000 cells per well) and transfected with $20 \mathrm{ng}$ pV16-PPAR $\gamma 2$ (WT or mutated) $20 \mathrm{ng}$ pM-MED1, $50 \mathrm{ng}$ pG5-luc (Promega) reporter plasmid and $6 \mathrm{ng}$ pRL-SV40 (Promega), in the presence of the Fugene HD transfection reagent (Promega), in accordance with the manufacturer's protocol. Luciferase activity was determined $48 \mathrm{~h}$ later, with the Dual-Glo ${ }^{\circledR}$ Luciferase Assay System (Promega), according to the manufacturer's instructions, and the results obtained were normalized with the Renilla luciferase signal obtained with the pRL-SV40 plasmid.

Immunoblotting. The 5637 and RT4 cell lysates were clarified by centrifugation. The protein concentration of the supernatants was determined with the BCA protein assay (Thermo Scientific). $10 \mu \mathrm{g}$ of proteins were resolved by SDS-PAGE in a $4-15 \%$ polyacrylamide gels, electrotransferred onto Biorad nitrocellulose membranes and analyzed by incubation with primary antibodies against PPAR $\gamma$ (Abcam \#ab41928, used at 1/1000) and $\beta$-actin (Sigma Aldrich \#A2228, used at $1 / 25,000)$. Horseradish peroxidase-conjugated anti-mouse IgG (Cell Signaling Technology \# 7074, used at 1/3000) was used as the secondary antibody. Protein loading was checked by staining the membrane with Amido Black after electroblotting. Uncropped scans of the western blot are supplied Supplementary Fig. 15

Real-time reverse transcription-quantitative PCR. Reverse transcription was performed with $1 \mu \mathrm{g}$ of total RNA, and a high-capacity cDNA reverse transcription kit (Applied Biosystems). cDNAs were amplified by PCR in a Roche real-time thermal cycler, with the Roche Taqman master mix (Roche) and Taqman probe/ primer pairs as follows:

PLIN2:

Forward primer-TCTGAATCAGCCATCAACTCAG;

Reverse primer-GTGCTGGCCACAGAATCC;

Roche Taqman probe no. 57

ACSL5:

Forward primer-TGTCCACTTCAGTCATGACATTCT;

Reverse primer-TCCAGTCCCCAGGTAATGTAA;

Roche Taqman probe no. 83 
FABP4:

Forward primer-GGATGATAAACTGGTGGTGGA; Reverse primer-CACAGAATGTTGTAGAGTTCAATGC

Roche Taqman probe no. 85

PPAR $\gamma$ and TBP were amplified by PCR in a Roche real-time thermal cycler

with Roche SYBR green master mix and primers, as follows:

PPAR $\gamma$ :

Forward primer-GCCCAAGTTTGAGTTTGCTG;

Reverse primer-TCAATGGGCTTCACATTCAGC;

TBP:

Forward primer-TTGCTGCGGTAATCATGAGG

Reverse primer-TTTTCTTGCTGCCAGTCTGG

We used the $4326322 E$ assays on demand for TBP (encompassing primers and

Taqman probes) purchased from Applied Life Technologies.

Relative gene expression was analyzed by the delta delta Ct method, with TBP as the reference.

Biochemistry. The sequences encoding the ligand-binding domains of the HishPPAR $\gamma$ (231-505) and His-mRXRa (228-467) receptors were inserted into pET15b. Point mutations were introduced into PPAR $\gamma$ with the QuikChange II XL Site-Directed Mutagenesis kit (Agilent), in accordance with the manufacturer's instructions.

The corresponding proteins were produced in Escherichia coli BL21 DE3 by overnight incubation at $22^{\circ} \mathrm{C}$ after induction with $1 \mathrm{mM}$ IPTG at an $\mathrm{OD}_{600}$ of $\sim 0.8$. Soluble proteins were purified by Ni-NTA chromatography followed by size exclusion chromatography on a Superdex 200 (GE) column equilibrated in $25 \mathrm{mM}$ Tris- $\mathrm{HCl}, \mathrm{pH} 8.0,200 \mathrm{mM} \mathrm{NaCl}, 5 \%$ glycerol, and $1 \mathrm{mM}$ TCEP. The proteins were concentrated to $3-6 \mathrm{mg} / \mathrm{ml}$ with an Amicon Ultra $10 \mathrm{kD}$ MWCO. Purity and homogeneity of all proteins were assessed by SDS and Native Page and for PPAR proteins by denaturing and native electrospray ionization mass spectrometry (Supplementary Figs. 3 and 4).

Crystallization, data collection, and structure refinement. Crystallization trials were performed with either apo protein or with the protein in complex with a threefold excess of GW1929 or a threefold excess of GW1929 and PGC1a peptide. The crystallization experiments were performed by sitting drop vapor diffusion at $290 \mathrm{~K}$, mixing equal volumes $(200 \mathrm{nl})$ of protein at $5 \mathrm{mg} / \mathrm{ml}$ and reservoir solution. For all crystal structures, the data were indexed and integrated with $\mathrm{XDS}^{36}$ and scaled with AIMLESS ${ }^{37,38}$. The structure was solved by molecular replacement in PHASER $^{39}$ and refined with PHENIX ${ }^{40}$ and BUSTER ${ }^{41}$ with TLS refinement, followed by iterative model building in $\mathrm{COOT}^{42}$.

Crystals of PPAR $\gamma$ M280I-GW1929-PGCla were grown in 20\% PEG 550 MME, 10\% PEG 20000, $30 \mathrm{mM}$ sodium fluoride, $30 \mathrm{mM}$ sodium bromide, $30 \mathrm{mM}$ sodium iodide, $0.1 \mathrm{M}$ sodium Hepes/MOPS pH 7.5, transferred to artificial mother liquor containing $15 \%$ glycerol and flash-cooled in liquid nitrogen. X-ray diffraction data were collected at PX1 beamline of the SOLEIL synchrotron with a wavelength of $0.979 \AA$. The final structure was refined to $R_{\text {work }}$ and $R_{\text {free }}$ values of $17.5 \%$ and $22.6 \%$, respectively, with excellent geometry $(97.99 \%$ of residues in favored region of the Ramachandran plot, $2.01 \%$ in the allowed region, and $0.0 \%$ outliers).

Crystals of PPAR $\gamma$ T475M-GW1929-PGC1a were grown in 25\% PEG 3350, $0.2 \mathrm{M}$ ammonium sulfate, $0.1 \mathrm{M}$ Bis-Tris $\mathrm{pH} 6.5$, transferred to artificial mother liquor containing $15 \%$ glycerol and flash-cooled in liquid nitrogen. X-ray diffraction data were collected at the ID23-1 beamline of ESRF with a wavelength of $0.9724 \AA$. The final structure was refined to $R_{\text {work }}$ and $R_{\text {free }}$ values of $15.9 \%$ and $19.88 \%$, respectively, with excellent geometry $(98.23 \%$ of residues in favored region of the Ramachandran plot, $1.77 \%$ in the allowed region, and $0.0 \%$ outliers).

Crystals of PPAR $\gamma$ T475M apo were grown in $0.5 \mathrm{M}$ ammonium sulfate, $0.1 \mathrm{M}$ PIPES $\mathrm{pH}$ 7, $0.9 \mathrm{M}$ disodium tartrate. Crystals were transferred to artificial mother liquor containing $15 \%$ glycerol and flash-cooled in liquid nitrogen. X-ray diffraction data were collected at the ID23-1 beamline of ESRF with a wavelength of $0.9724 \AA$. The final structure was refined to $R_{\text {work }}$ and $R_{\text {free }}$ values of $19.5 \%$ and $23.48 \%$, respectively, with good geometry $(96.05 \%$ of residues in favored region of the Ramachandran plot, $3.77 \%$ in the allowed region, and $0.19 \%$ outliers).

Crystals of PPAR $\gamma$ WT-GW1929-PGCla were grown in 20\% PEG 5000 MME, $0.1 \mathrm{M}$ Bis-Tris pH 6.5. Crystals were transferred to artificial mother liquor containing $15 \%$ glycerol and flash-cooled in liquid nitrogen. X-ray diffraction data were collected at the ID30A beamline of ESRF with a wavelength of $0.968 \AA$. The final structure was refined to $R_{\text {work }}$ and $R_{\text {free }}$ values of $21.9 \%$ and $25.2 \%$, respectively, with excellent geometry $(96.53 \%$ of residues in favored region of the Ramachandran plot, $3.47 \%$ in the allowed region, and $0.00 \%$ outliers).

Crystals of PPAR $\gamma$ I290M-GW1929 complex were grown in 25\% PEG 3350, $0.2 \mathrm{M} \mathrm{NaCl}, 0.1 \mathrm{M}$ Hepes pH 7.5 and were transferred to artificial mother liquor containing 35\% PEG 3350 before flash-cooling. X-ray diffraction data were collected at the ID23-1 beamline of ESRF with a wavelength of $0.9724 \AA$. The final structure was refined to $R_{\text {work }}$ and $R_{\text {free }}$ values of $16.52 \%$ and $20.89 \%$, respectively, with excellent geometry ( $98.54 \%$ of residues in favored region of the Ramachandran plot, $1.46 \%$ in the allowed region, and $0.00 \%$ outliers).

Data collection and refinement statistics are provided in Supplementary Table 5. GW1929 and side chains of the mutated residues could be modeled with confidence in all chains and all complexes as shown into the Polder omit maps ${ }^{43}$ displaying reduced model bias and exclusion of solvent molecules (Supplementary Figs. 9A and 12A). All structural figures were prepared with PyMOL (www.pymol.org/).

Molecular dynamics simulations. hPPAR $\gamma$ PDB:2PRG ${ }^{20}$ was used for all calculations. For the WT holo form, we used chain A, the ligand and coactivator peptide segment from the crystal structure. For the WT apo form, we used the same structure, but we removed the ligand and the coactivator peptide from the structure. For the corresponding mutant forms, the point mutation was built into this experimental crystal structure with CHARMM program version $\mathrm{c} 37 \mathrm{~b} 1^{44}$. All forms were subjected to the PROPKA program ${ }^{45}$ to determine the protonation states of titratable residues. These calculations indicated that the $\mathrm{H} 217, \mathrm{H} 266$, $\mathrm{H} 323$, His449, and $\mathrm{H} 466$ residues of chain A were protonated on the $\mathrm{N} \varepsilon$, but that $\mathrm{H} 425$ could be considered doubly protonated. We modified the PDB file accordingly to incorporate the correct protonation states. Hydrogen atom positions were positioned with the HBUILD ${ }^{46}$ module of the CHARMM program. The parameters for rosiglitazone were determined in a previous study ${ }^{47}$, MD simulations were performed with NAMD software ${ }^{48}$ and the CHARMM all-atom force field, version $36^{49}$. Crystal water molecules associated with the hPPAR $\gamma$ protein were retained. Energy minimization was performed for the protein, in 700 steps, with the steepest descent (SD) algorithm in CHARMM software. Non-bonded interactions were truncated at a $14 \AA$ cutoff distance, using switch and shift functions for van der Waals and electrostatic forces, respectively. The protein was then placed in a cubic TIP3P explicit water box of dimensions $80 \times 80 \times 80 \AA$. Chloride and sodium counter ions were added to the water box to neutralize the charge of the system and additional ions were added to yield a final concentration of $0.15 \mathrm{M}$. MD simulations were initiated with two rounds of energy minimization and heating while the atomic coordinates of the protein complex were fixed. In the first phase, water was minimized by 1000 steps of conjugate gradient (CG) and then heated to $600 \mathrm{~K}$. In the second phase, the water was minimized by 250 steps of CG, followed by heating to $300 \mathrm{~K}$. The constraints on the protein were then removed and the entire system was subjected to energy minimization through 2000 steps of CG and subsequent heating to $300 \mathrm{~K}$. The system was then equilibrated for $150 \mathrm{ps}$. With a $1 \mathrm{fs}$ time step, the production phase extended over a total of $107 \mathrm{~ns}$. This protocol was used for all forms studied. The root-mean-square deviation (RMSD) and root-mean-square fluctuation (RMSfl) were calculated using the CHARMM program and the coactivator peptide binding energies were estimated by an MM/GBSA procedure ${ }^{25}$ using the CHARMM program combined with in-house programs.

Microscale thermophoresis. Measurements were performed with a Monolith NT.115 instrument (NanoTemper Technologies GmbH, Munchen, Germany). The PPAR $\gamma$ complexes were prepared in $20 \mathrm{mM}$ Tris $\mathrm{pH} 8.0,200 \mathrm{mM} \mathrm{NaCl}, 1 \mathrm{mM}$ TCEP, $0.05 \%$ Tween 20 . Each measurement consists of 16 reaction mixtures where the fluorescent-labeled peptide concentration was constant $(150 \mathrm{nM})$ and serial dilutions of PPAR $\gamma$ LBD from a concentration of $100 \mu \mathrm{M}$ down to $2 \mathrm{nM}$. Measurements were made with standard glass capillaries (Nanotemper) at $25^{\circ} \mathrm{C}$, at $30 \%$ LED excitation and $40-80 \%$ MST power, with a laser-on time of $30 \mathrm{~s}$ and a laser-off time of $5 \mathrm{~s}$. NanoTemper Analysis 2.2.4 software was used to fit the data and to determine the $K_{\mathrm{d}}$

Analytical ultracentrifugation. Sedimentation velocity experiments were performed at $4^{\circ} \mathrm{C}$, with a Beckman Optima XL-A analytical ultracentrifuge equipped with absorbance optics and an An50-Ti rotor in a buffer containing $20 \mathrm{mM}$ Tris, $\mathrm{pH}$ 8.0, $300 \mathrm{mM} \mathrm{NaCl}, 5 \mathrm{mM}$ Glycerol, $1 \mathrm{mM}$ TCEP. For this analysis, the heterodimer was formed by mixing the monomeric PPAR $\gamma$ and RXRa proteins into a 1:1 molar ratio. Protein concentrations were in the range of 0.8 to $0.9 \mathrm{mg} / \mathrm{ml}$. The sedimentation velocity analysis was conducted at $165,500 \times g$. Values were normalized to standard conditions by correcting for buffer density and viscosity. Sedimentation coefficient distributions were calculated from the sedimentation velocity data using the SEDFIT software program (www.analyticalultracentrifugation.com).

Surface plasmon resonance. The SPR measurements were performed by Biacore T100 sensitivity enhanced T200 equipment (GE Healthcare) using CM5 series S sensor chip. The RXR $\alpha$ LBD monomer was immobilized on the chip surface using standard amino-coupling protocol in $10 \mathrm{mM} \mathrm{Na}$-acetate buffer $\mathrm{pH}$ 5.5. The resulting immobilized RXR was in the range of 400-500 response units. The running buffer was $50 \mathrm{mM}$ Tris $\mathrm{pH} 7.5,150 \mathrm{mM} \mathrm{NaCl}, 1 \mathrm{mM}$ TCEP, $0.005 \%$ Tween 20 and for regeneration $1 \mathrm{M}$ sodium chloride solution was used. Interactions of the RXRa LBD with the LBDs of PPAR $\gamma$ WT, T475M and M280I mutants were analyzed in the manner of dose response using twofold dilution series of PPAR $\gamma$ LBDs ranging from 1 to $250 \mathrm{nM}$. The association phase was $120 \mathrm{~s}$ and the dissociation phase was $120 \mathrm{~s}$. After subtracting the reference and buffer signal, the data were fit to a steady state binding model and 1:1 kinetic model to define the $K_{\mathrm{D}}$ and $k_{\text {off }}$ using the Biacore T200 Evaluation software (GE Healthcare).

Mass spectrometry analysis. Prior to mass spectrometry analysis, PPAR $\gamma$ and all the different mutant proteins were buffer exchanged against $200 \mathrm{mM}$ of ammonium 
acetate at $\mathrm{pH} 6.8$, using five cycles of concentration/dilution with a microconcentrator (Vivaspin, 10-kD cutoff, Sartorius, Göttingen, Germany). All the samples were diluted either in $\mathrm{H}_{2} \mathrm{O} / \mathrm{ACN} / \mathrm{HCOOH}$ (denaturing MS conditions) or in $200 \mathrm{mM} \mathrm{AcONH}_{4}$ (native MS conditions) to a final concentration of $5 \mu \mathrm{M}$ and infused with an automated chip based nanoelectrospray device (Triversa Nanomate, Advion Bioscience, Ithaca, USA) operating in the positive ion mode, coupled to a Synapt G2 HDMS mass spectrometer (Waters, Manchester, UK). Under denaturing mass spectrometry conditions, the backing pressure, the cone voltage and the extraction voltage of the mass spectrometer were set to $1.47 \mathrm{mbar}$, $20 \mathrm{~V}$, and $4 \mathrm{~V}$, respectively. The trap cell was operating under a constant $\mathrm{Ar}$ pressure of $9 \times 10^{-3} \mathrm{mbar}(2 \mathrm{ml} / \mathrm{min})$. The voltage within the trap cell and the trap DC bias were set to $7 \mathrm{~V}$ and $2 \mathrm{~V}$, respectively. In this case, the traveling wavebased helium cell and ion mobility cell were used as ion guides under $1.2 \times 10^{-4}$ mbar and $8 \times 10^{-5}$ mbar, respectively. In the transfer cell, ions were transmitted using an acceleration voltage of $7 \mathrm{~V}$ under a constant pressure of $10^{-6}$ mbar. The pressure within the time of flight analyzer was kept at $6.4 \times 10^{-7}$ mbar. For native mass spectrometry analysis, the cone voltage, the extraction voltage and the backing pressure of the mass spectrometer were set to $20 \mathrm{~V}, 5 \mathrm{~V}$, and 6 mbar, respectively, in order to improve the transmission of the "native-like" molecular ions and avoiding ion heating. Ions were efficiently trapped with a constant Ar flow rate of $4.5 \mathrm{ml} / \mathrm{min}$ (leading to a final pressure in the trap cell of $8.8 \times 10^{-3} \mathrm{mbar}$ ) and $4 \mathrm{~V}$ and then they were pulse driven with a trap DC bias of $2 \mathrm{~V}$. During mass spectrometry analysis, the pressure within the helium cell and ion mobility cell were kept at $1.2 \times 10^{-4}$ and $8 \times 10^{-5}$ mbar. Ions were transferred from the ion mobility cell to the analyzer by applying a constant voltage of $2 \mathrm{~V}$ under a constant pressure of $10^{-6} \mathrm{mbar}$ in the transfer cell. Finally, ions were analyzed within the time of flight analyzer under high vacuum conditions $\left(6.3 \times 10^{-7} \mathrm{mbar}\right)$ in order to ensure highly accurate mass measurements. Mass spectra recorded with Synapt G2 platform were analyzed with MassLynx 4.1 (Waters, Manchester, UK).

Native IM-MS. Ion mobility experiments were performed on the Synapt G2 HDMS (Waters, Manchester, UK). The backing pressure, the cone voltage and the extraction cone was set to $6 \mathrm{mbar}, 40 \mathrm{~V}$, and $5 \mathrm{~V}$, respectively. The traveling wavebased ion trap was filled with a continuous Ar flow of $6 \mathrm{ml}$ per min and the trap collision energy was set to $4 \mathrm{~V}$. Ions were pulse-driven with a trap DC bias of $60 \mathrm{~V}$ and subsequently separated in the TWIMS (Traveling Wave Ion Mobility Spectrometry) cell under a constant N2 pressure of $5.36 \mathrm{mbar}$ (N2 flow rate of $25 \mathrm{ml}$ per $\mathrm{min})$. The IM wave velocity and height were set to $1250 \mathrm{~m} / \mathrm{s}$, and $40 \mathrm{~V}$, respectively. The other mass spectrometer parameters not described in this section remained the same as those used during the native mass spectrometry analysis. IM data were calibrated as described elsewhere. Briefly, three charge states of two external calibrants (Cytochrome $C$ and $\beta$-lactoglobuline) were used to determine the rotationally averaged collision cross section (CCS) of the ion of interest. MassLynx 4.1 was used to perform IM data interpretation. Reported TWCCSN2 values correspond to the average TWCCSN2 measurement performed in triplicate under the same experimental conditions.

Collision-induced unfolding (CIU) experiments. Prior to IM separation, ions were progressively activated in the trap cell by increasing the acceleration voltage in $5 \mathrm{~V}$ steps from 0 to $60 \mathrm{~V}$. Ion mobility data at each individual voltage were acquired during $1 \mathrm{~min}$, and finally compiled to give rise to the CIU fingerprints. The arrival time distribution (ATD) of each individual charge state of the protein complexes were extracted with the open-source CIU-Suite software ${ }^{50}$. CIU experiments have been performed in triplicate for each individual complex leading to a standard deviation lower than $4 \%$, calculated with the CIUSuite-Stat module included in the CIU-Suite package.

HDX-MS experiments. HDX of PPAR $\gamma$ WT, M280I and T475M proteins were carried out with and without PGC1 $\alpha$ peptide, with 1:3 concentration ratios in $20 \mathrm{mM}$ Tris ( $\mathrm{pH} 8.0$ ), $200 \mathrm{mM} \mathrm{NaCl}, 5 \%$ glycerol. Samples were incubated for a range of exchange times ( $0 \mathrm{~min}, 0.5 \mathrm{~min}, 2 \mathrm{~min}, 10 \mathrm{~min}, 30$ and $60 \mathrm{~min}$ ) in $95 \%$ of deuterated buffer $(20 \mathrm{mM}$ Tris $(\mathrm{pH} 8.0), 200 \mathrm{mM} \mathrm{NaCl})$ before quenching the exchange reaction by adding a quench solution $(100 \mathrm{mM}$ glycine, 2 $\mathrm{M} \mathrm{GdHCl}, \mathrm{pH} 2.2)$ at $1{ }^{\circ} \mathrm{C}$ during $30 \mathrm{~s}$. Protein digestion of quenched samples $(120$ pmol) was then performed through a pepsin-immobilized cartridge in $0.1 \%$ aqueous formic acid solution at a $200 \mu \mathrm{l} / \mathrm{min}$. The digested peptides were then trapped on UPLC pre-column (ACQUITY UPLC BEH C18 VanGuard pre-column, 2.1 mm I.D. $\times 5 \mathrm{~mm}, 1.7 \mu \mathrm{M}$ particle diameter, Waters) and separated on UPLC column (ACQUITY UPLC BEH C18, $1.0 \mathrm{~mm}$ I.D. $\times 100 \mathrm{~mm}, 1.7 \mu \mathrm{M}$ particle diameter, Waters) at $0{ }^{\circ} \mathrm{C}$. Preparation and injection of the samples were automatically conducted by the CTC PAL robot (Leap Technologies, Zwingen, Switzerland), while chromatographic step was carried out on Acquity UPLC system with HDX technology (Waters, Milford, MA, USA). Mass spectrometry measurements were acquired with Synapt G2Si HDMS (Waters) with electrospray ionization, using data-independent acquisition mode $\left(\mathrm{MS}^{\mathrm{E}}\right)$ over an $\mathrm{m} / \mathrm{z}$ range of 50-2000 and with $100 \mathrm{fmol} / \mu \mathrm{l} \mathrm{Glu-FibrinoPeptide} \mathrm{as} \mathrm{lock-mass} \mathrm{correction.}$ HDX experiments were realized in triplicate for each time point. Peptide identification was performed using ProteinLynx Global Server 2.5.3 (Waters) with a custom protein sequence library, where peptide and fragment tolerances were automatically adjusted by PLGS, while oxidized methionine was set as variable modification. Deuterium uptakes for all identified peptides were checked and validated manually using DynamX 3.0 (Waters): only peptides-with a length range of 5-25 residues-identified in all replicates were kept with a minimum products per amino acid of 0.3 . Only one charge state was kept for each peptide and deuterium uptakes were not corrected for back-exchange, representing as relative. HDX-MS results were statistically validated using Mixed-Effects Model for HDX experiments (MEMHDX) ${ }^{29}$, using Wald tests, where statistical significance thresholds were set to 0.01 .

Reporting summary. Further information on experimental design is available in the Nature Research Reporting Summary linked to this article.

\section{Data availability}

Atomic coordinates and related structure factors have been deposited in the Protein Data Bank with accession codes: 6FZY (PPAR $\gamma$ T475M apo), 6FZF (PPAR $\gamma$ T475M-GW1929-PGC1 $\alpha$ ), 6FZJ (PPAR $\gamma$ M280I-GW1929-PGC1 $\alpha$ ), 6FZG (PPAR $\gamma$ I290M-GW1929) and 6FZP (PPAR $\gamma-$ GW1929-PGC1a). The source data underlying Figs. 1-3 and Supplementary Figs. 1, 13 and 14 are provided as a Source Data file. A reporting summary for this Article is available as a Supplementary Information file. All other data supporting the findings of this study are available from the corresponding authors on reasonable request.

Received: 16 March 2018 Accepted: 18 December 2018

Published online: 16 January 2019

\section{References}

1. Tontonoz, P. \& Spiegelman, B. M. Fat and beyond: the diverse biology of PPARgamma. Annu. Rev. Biochem. 77, 289-312 (2008).

2. Ahmadian, M. et al. PPARgamma signaling and metabolism: the good, the bad and the future. Nat. Med. 19, 557-566 (2013).

3. Varley, C. L. et al. Role of PPARgamma and EGFR signalling in the urothelial terminal differentiation programme. J. Cell. Sci. 117, 2029-2036 (2004).

4. Peters, J. M., Shah, Y. M. \& Gonzalez, F. J. The role of peroxisome proliferatoractivated receptors in carcinogenesis and chemoprevention. Nature Rev. Cancer 12, 181-195 (2012).

5. Lee, J. J., Drakaki, A., Iliopoulos, D. \& Struhl, K. MiR-27b targets PPARgamma to inhibit growth, tumor progression and the inflammatory response in neuroblastoma cells. Oncogene 31, 3818-3825 (2012).

6. Ahmad, I. et al. Sleeping Beauty screen reveals Pparg activation in metastatic prostate cancer. Proc. Natl Acad. Sci. USA 113, 8290-8295 (2016).

7. Biton, A. et al. Independent component analysis uncovers the landscape of the bladder tumor transcriptome and reveals insights into luminal and basal subtypes. Cell Rep. 9, 1235-1245 (2014).

8. Goldstein, J. T. et al. Genomic activation of PPARG reveals a candidate therapeutic axis in bladder cancer. Cancer Res. 77, 6987-6998 (2017).

9. Cancer Genome Atlas Research, N. Comprehensive molecular characterization of urothelial bladder carcinoma. Nature 507, 315-322 (2014).

10. Robertson, A. G. et al. Comprehensive molecular characterization of muscleinvasive bladder. Cell 171, 540-556 (2017).

11. Hedegaard, J. et al. Comprehensive transcriptional analysis of early-stage urothelial carcinoma. Cancer Cell 30, 27-42 (2016).

12. Choi, W. et al. Identification of distinct basal and luminal subtypes of muscleinvasive bladder cancer with different sensitivities to frontline chemotherapy. Cancer Cell 25, 152-165 (2014).

13. Korpal, M. et al. Evasion of immunosurveillance by genomic alterations of PPARgamma/RXRalpha in bladder cancer. Nat. Commun. 8, 103 (2017).

14. Halstead, A. M. et al. Bladder-cancer-associated mutations in RXRA activate peroxisome proliferator-activated receptors to drive urothelial proliferation. eLife 6, e30862 (2017)

15. Van Allen, E. M. et al. Somatic ERCC2 mutations correlate with cisplatin sensitivity in muscle-invasive urothelial carcinoma. Cancer Discov. 4 1140-1153 (2014)

16. Kim, J. B., Wright, H. M., Wright, M. \& Spiegelman, B. M. ADD1/SREBP1 activates PPARgamma through the production of endogenous ligand. Proc. Natl Acad. Sci. USA 95, 4333-4337 (1998).

17. Liberato, M. V. et al. Medium chain fatty acids are selective peroxisome proliferator activated receptor (PPAR) $\gamma$ activators and pan-PPAR partial agonists. PLoS ONE 7, e36297 (2012).

18. Brown, K. K. et al. A novel N-aryl tyrosine activator of peroxisome proliferator-activated receptor-gamma reverses the diabetic phenotype of the Zucker diabetic fatty rat. Diabetes 48, 1415-1424 (1999). 
19. Berman, H. M. et al. The protein data bank. Nucleic Acids Res. 28, 235-242 (2000).

20. Nolte, R. T. et al. Ligand binding and co-activator assembly of the peroxisome proliferator-activated receptor-gamma. Nature 395, 137-143 (1998).

21. Ohashi, M., Oyama, T. \& Miyachi, H. Different structures of the two peroxisome proliferator-activated receptor gamma (PPARgamma) ligandbinding domains in homodimeric complex with partial agonist, but not full agonist. Bioorg. Med. Chem. Lett. 25, 2639-2644 (2015).

22. Chrisman, I. M. et al. Defining a conformational ensemble that directs activation of PPAR $\gamma$. Nat. Commun. 9, 1794 (2018).

23. Gampe, R. T. et al. Asymmetry in the PPARgamma/RXRalpha crystal structure reveals the molecular basis of heterodimerization among nuclear receptors. Mol. Cell 5, 545-555 (2000).

24. Hamuro, Y. et al. Hydrogen/deuterium-exchange (H/D-Ex) of PPARgamma LBD in the presence of various modulators. Protein Sci. 15, 1883-1892 (2006).

25. Kollman, P. A. et al. Calculating structures and free energies of complex molecules: combining molecular mechanics and continuum models. Acc. Chem. Res. 33, 889-897 (2000).

26. Genest, D. et al. Ligand-escape pathways from the ligand-binding domain of PPARgamma receptor as probed by molecular dynamics simulations. Eur. Biophys. J. 37, 369-379 (2008).

27. Hughes, T. S. et al. An alternate binding site for PPARgamma ligands. Nat. Commun. 5, 3571 (2014).

28. Capelli, D. et al. Structural basis for PPAR partial or full activation revealed by a novel ligand binding mode. Sci. Rep. 6, 34792 (2016).

29. Hourdel, V. et al. MEMHDX: an interactive tool to expedite the statistical validation and visualization of large HDX-MS datasets. Bioinformatics 32, 3413 (2016).

30. Raman, P. \& Koenig, R. J. Pax-8-PPAR-gamma fusion protein in thyroid carcinoma. Nat. Rev. Endocrinol. 10, 616-623 (2014).

31. Ristow, M., Muller-Wieland, D., Pfeiffer, A., Krone, W. \& Kahn, C. R. Obesity associated with a mutation in a genetic regulator of adipocyte differentiation. N. Engl. J. Med. 339, 953-959 (1998).

32. Robinson, D. R. et al. Activating ESR1 mutations in hormone-resistant metastatic breast cancer. Nat. Genet. 45, 1446-1451 (2013).

33. Shukla, G. C., Plaga, A. R., Shankar, E. \& Gupta, S. Androgen receptor-related diseases: what do we know? Andrology 4, 366-381 (2016).

34. Semple, R. K., Chatterjee, V. K. \& O'Rahilly, S. PPAR gamma and human metabolic disease. J. Clin. Invest. 116, 581-589 (2006).

35. Eelen, G. et al. Superagonistic action of 14-epi-analogs of 1,25dihydroxyvitamin D explained byvitaminDreceptor-coactivatorinteraction. Mol. Pharmacol. 67, 1566-1573 (2005).

36. Kabsch, W. Xds. Acta Crystallogr. D Biol Crystallogr. 66, 125-132 (2010).

37. Evans, P. Scaling and assessment of data quality. Acta Crystallogr. D Biol. Crystallogr. 62, 72-82 (2006).

38. Collaborative Computational Project, N. The CCP4 suite: programs for protein crystallography. Acta Crystallogr. D Biol. Crystallogr. 50, 760-763 (1994).

39. McCoy, A. J., Grosse-Kunstleve, R. W., Storoni, L. C. \& Read, R. J. Likelihoodenhanced fast translation functions. Acta Crystallogr. D Biol. Crystallogr. 61, 458-464 (2005).

40. Adams, P. D. et al. PHENIX: a comprehensive Python-based system for macromolecular structure solution. Acta Crystallogr. D Biol. Crystallogr. 66 , 213-221 (2010).

41. Smart, O. S. et al. Exploiting structure similarity in refinement: automated NCS and target-structure restraints in BUSTER. Acta Crystallogr. D Biol. Crystallogr. 68, 368-380 (2012).

42. Emsley, P. \& Cowtan, K. Coot: model-building tools for molecular graphics. Acta Crystallogr. D Biol. Crystallogr. 60, 2126-2132 (2004).

43. Liebschner, D. et al. Polder maps: improving OMIT maps by excluding bulk solvent. Acta Crystallogr. D Struct. Biol. 73, 148-157 (2017).

44. Brooks, B. R. et al. CHARMM: the biomolecular simulation program. J. Comput. Chem. 30, 1545-1614 (2009).

45. Olsson, M. H., Sondergaard, C. R., Rostkowski, M. \& Jensen, J. H. PROPKA3: Consistent Treatment of Internal and Surface Residues in Empirical pKa Predictions. J. Chem. Theory Comput. 7, 525-537 (2011).

46. Brunger, A. T. \& Karplus, M. Polar hydrogen positions in proteins: empirical energy placement and neutron diffraction comparison. Proteins 4, 148-156 (1988).

47. Fidelak, J. et al. Dynamic correlation networks in human peroxisome proliferator-activated receptor-gamma nuclear receptor protein. Eur. Biophys. J. 39, 1503-1512 (2010).

48. Phillips, J. C. et al. Scalable molecular dynamics with NAMD. J. Comput. Chem. 26, 1781-1802 (2005).

49. MacKerell, A. D. et al. All-atom empirical potential for molecular modeling and dynamics studies of proteins. J. Phys. Chem. B 102, 3586-3616 (1998).
50. Eschweiler, J. D., Rabuck-Gibbons, J. N., Tian, Y. \& Ruotolo, B. T. CIUSuite: a quantitative analysis package for collision induced unfolding measurements of gas-phase protein ions. Anal. Chem. 87, 11516-11522 (2015).

51. Osz, J. et al. Solution structures of PPARgamma2/RXRalpha complexes. PPAR Res. 2012, 701412 (2012)

\section{Acknowledgements}

This work was supported by a grant from Ligue Nationale Contre le Cancer (C.K., L.C.-T., E.G., R.Z., S.R., Y.N., Y.A., F.R., I.B.-P.) as an associated team (Equipe labellisée), the "Carte d'Identité des Tumeurs" program initiated, developed and funded by Ligue Nationale Contre le Cancer, by a "PL-Bio" project funded by INCa (2016-146), the French Ministry of Education and Research, the CNRS, and the Institut Curie. The project was also supported by the Centre National de la Recherche Scientifique, the Institut National de la Santé et de la Recherche Médicale and the University of Strasbourg. We acknowledge the support and the use of resources of the French Infrastructure for Integrated Structural Biology FRISBI ANR-10-INBS-05, the Instruct-ERIC and the French Proteomic Infrastructure ProFI ANR-10-INBS-08-03. We would like to thank the staff of Proxima 1 at SOLEIL as well as of ID23 and ID30A at ESRF for assistance in using the beamlines. We also thank Alastair McEwen (IGBMC) for help in X-ray data collections and structural refinement and Camille Kostmann (IGBMC) for assistance in SPR. We would like to thank Simone Benhamou (IGR) and Thierry Lebret (Foch Hospital) for their help in setting up the CIT series of bladder tumors. We thank GIS IBiSA and Région Alsace for financial support in purchasing a Synapt G2 HDMS instrument. We acknowledge the Strasbourg University High Performance Computing Center and their staff for providing access to computing resources and for their support. We also acknowledge GENCI (Grand Équipement National de Calcul Intensif) for computing resources and the Equipex Equip@Meso project (Programme Investissements d'Avenir). MB was supported by a fellowship from the Région Alsace. OA-H acknowledges the IdeX program of the University of Strasbourg for funding his postdoctoral fellowship.

\section{Author contributions}

N.R., R.H.S. F.R. and I.B.-P. designed the study. H.L., C.B. and T.M. provided and characterized the tumors from the Strasbourg cohort. C.K., R.Z. and E.G. sequenced PPARG and RXRA and analyzed the data together with L.C.-T., A.K. and A.d.R. who carried out the bioinformatics analysis. A.K., A.d.R. and Y.A. classified the tumors according to pathological status and to the different subgroups identified by the TCGA. C.K., L.C.-T., F.D., S.R., Y.N. and I.B.-P. performed the functional studies and analyzed the data. J.O., W.Z., C.P.-I. and S.H.-B. performed the biochemical, biophysical, and structural studies, and J.O. and N.R. analyzed the data. S.V. and K.A. performed the molecular dynamics, and A.D. and R.H.S. analyzed the MD data. O.A.H. and M.B. performed the mass spectrometry experiments, O.A.H., M.B. and S.C. analyzed the mass spectrometry data. N.R., C.K., L.C.-T., R.H.S., F.R. and I.B.-P. wrote the manuscript. All authors made comments on the manuscript.

\section{Additional information}

Supplementary Information accompanies this paper at https://doi.org/10.1038/s41467018-08157-y.

Competing interests: The authors declare no competing interests.

Reprints and permission information is available online at http://npg.nature.com/ reprintsandpermissions/

Journal peer review information: Nature Communications thanks the anonymous reviewers for their contribution to the peer review of this work. Peer reviewer reports are available.

Publisher's note: Springer Nature remains neutral with regard to jurisdictional claims in published maps and institutional affiliations.

(c) (i) Open Access This article is licensed under a Creative Commons cc) Attribution 4.0 International License, which permits use, sharing, adaptation, distribution and reproduction in any medium or format, as long as you give appropriate credit to the original author(s) and the source, provide a link to the Creative Commons license, and indicate if changes were made. The images or other third party material in this article are included in the article's Creative Commons license, unless indicated otherwise in a credit line to the material. If material is not included in the article's Creative Commons license and your intended use is not permitted by statutory regulation or exceeds the permitted use, you will need to obtain permission directly from the copyright holder. To view a copy of this license, visit http://creativecommons.org/ licenses/by/4.0/.

(C) The Author(s) 2019 